\title{
Benefit of the reactive extrusion in the course of polyhydroxyure- thane synthesis by aminolysis of cyclic carbonates
}

\author{
Fiona Magliozzi1 ${ }^{1}$, Guillaume Chollet ${ }^{2}$, Etienne Grau ${ }^{1}$ and Henri Cramail ${ }^{1, *}$ \\ ${ }^{1}$ Univ. Bordeaux, CNRS, Bordeaux INP, LCPO, UMR 5629, F-3360o, Pessac, France \\ ${ }^{2}$ ITERG, 11 Rue Gaspard Monge, 33610 Canéjan France
}

\begin{abstract}
Replacing toxic isocyanates and petro-based reactants currently employed for polyurethanes (PUs) synthesis is nowadays a major concern. Among all the possible routes to isocyanate-free PUs, aminolysis of cyclic carbonates, leading to polyhydroxyurethanes (PHUs) seems to be one of the most promising pathways. Herein, we discuss the added value brought by the reactive extrusion process on PHU synthesis. In order to evaluate the benefit and the versatility of the process, three different bis-cyclic carbonates exhibiting different reactivity (Diglycerol Dicarbonate, Seb-bCC-ester and one bisCC containing amide function, Und-6DA-bisCC), were tested in the course of polymerization with different diamines. The PHUs synthesized through reactive extrusion were then compared with the ones obtained through classical bulk polymerizations in terms of kinetics, PHU molar masses and side reactions, clearly demonstrating the benefit of the extrusion process.
\end{abstract}

KEYWORDS : Polyhydroxyurethanes, reactive extrusion, solvent-free polymerizations, biobased biscyclic carbonates, process improvement

First developed in the 1930s by Otto Bayer ${ }^{1}$, polyurethanes (PUs) are nowadays specialty polymers found in many markets, such as coatings, paints, elastomers, adhesives and especially, foams. Nonetheless, the current industrial 'polyol route' for the synthesis of conventional polyurethanes suffers from the use of toxic isocyanates, these latter made from even more hazardous phosgene gas. Despite the secured industrial setups to handle such reactants, their substitution in the PUs industry has become today a real question. ${ }^{2}$ Aiming at investigating safer alternatives, many research groups have, in recent years, been exploring new routes to non-isocyanates PUs, namely NIPUs.3 Among all these strategies, one of the most promising routes appears to be the polyaddition of cyclic carbonates (CC) with pluri-amines, through aminolysis reaction.

In addition, the combination of fossil resources depletion together with the need for isocyanate replacement, push scientists to develop bio-based monomers from renewable resources, including vegetable oils 4,5 , isosorbide ${ }^{6}$, sugars 7 , terpenes $^{8}$ and ligning. In this context, a large palette of cyclic carbonates has been produced from fully bio-based synthons. ${ }^{10-13}$
Most of the cyclic carbonates developed to form PHUs are 5membered cyclic carbonates, 5 -CCs. ${ }^{13-18}$ In a lesser extent, some PHUs can be also prepared from 6- to 8-membered cyclic carbonates ${ }^{19-26}$. These larger carbonate rings exhibit a higher reactivity in comparison to the 5 -CCs, due to their higher ring strain, but suffer from a lower stability at storage. In order to overcome the rather low reactivity of ${ }_{5} \mathrm{CCs}$, the introduction of functional groups exhibiting a withdrawing electronic effect in alpha or beta position of the carbonate ring is reported to increase its reactivity towards aminolysis. These so-called 'activated carbonates', first developed by Tomita et $a l .{ }^{27}$, were investigated by many research groups. ${ }^{28-31}$ In this course, we recently designed biscarbonates from fatty acid substrates bearing either ether, ester or thio-ester linkages, in beta position of the carbonate ring. ${ }^{29}$

Even if the polyaddition between CCs and pluri-amines seems to be the most promising route to NIPUs, such synthetic pathway still suffers from some drawbacks. As a general trend, notably with 5-CCs, their bulk polymerization exhibits a kinetic which is much slower in comparison to the 'polyol route', the reactive functions conversion is incomplete and some side reactions can occur with time. An important consequence of these polymerization features is generally the formation of relatively low molar mass PHUs. ${ }^{12,32,33}$ All these limitations are preventing the CC / amine route to NIPUs to be competitive with the current industrial PU process and thus still requires improvements that we aim at addressing in this work.

Indeed, intending to better control the monomer diffusion and overcome the viscosity issue generally encountered in bulk polymerization, we have been investigating the reactive extrusion process of bisCCs and diamines to evaluate the effect of such process on the kinetic profiles and PHUs features (molar masses and side reactions notably). The implementation of this process has also made possible to handle high melting point biscarbonates, which are challenging to polymerize in conventional bulk polymerization. Indeed, the reactive extrusion process enables a high shearing rate that prevents the use of high temperatures and, of course, solvents. Besides, the process presents the main advantage to significantly reduce the reaction times from days to hours, as short polymerization times and fast molar mass build-up are two crucial criteria for a competitive technical process. The literature data regarding the synthesis of NIPUs through reactive extrusion is rather scarce. Mulhaupt and coll. were the first to 
report NIPU synthesis through this process. In 2017, this team reported the polymerization of erythritol biscarbonate and different rigid and flexible diamines, in the presence of DABCO as catalyst. 34 Interestingly, the authors demonstrated that addition of Irganox 1010 and Irgafos 168, known as antioxidants to prevent degradation of polymers, could lead to higher molecular weights PHUs. More recently, the authors reported the polymerization of different bis-cyclic carbonates through reactive extrusion process, with 1.12 diaminododecane as a co-monomer, to design semi-crystalline PHUs, suitable for $3 \mathrm{D}$-printing applications. 35

In this study, a series of bio-based biscyclic carbonates, i.e. one ether-activated namely DGDC (Diglycerol Dicarbonate) for which the synthesis and the use for PHUs synthesis has been already described $36-39$, a second ester-activated, Seb-bCC-ester,(bis((2-oxo-1,3-dioxolan-4-yl)methyl) decanedioate), obtained from Glycerol Carbonate, and a third, non-activated and containing amide linkages, Und-6DA-bisCC, were reacted with the commercial diamines, 1.4-diaminobutane (4DA), 1.6diaminohexane (6DA), 1.10-diaminodecane (10DA), 1.12-diaminododecane (12DA), m-Xylylenediamine (xy-DA), and LLysine, by reactive extrusion. The polyhydroxyurethanes soformed were analyzed in terms of molecular structures, dimensions and thermal properties.

\section{EXPERIMENTAL SECTION}

\section{Materials}

The reactants 1.4-diaminobutane, 1.10-diaminodecane, 1.12-diaminododecane, m-Xylylenediamine, diglycerol, were purchased from TCI, Europe. $\mathrm{NaHCO}_{3}$, Anhydrous $\mathrm{MgSO}_{4}$ and Triethylamine, TBABr (tetra butyl amonium bromide), $\mathrm{m}$ CPBA (meta-chloroperbenzoic acid), and L-Lysine were ordered from Merck. Sodium methoxide (NaMeO) was provided by Alfa Aesar. Dimethylcarbonate (DMC, extra dry, 99\%), Sebacoyl Chloride and 1.6-diaminohexane were obtained from Acros. Solvents like DMF (for chromatography), dichloromethane (DCM), Methanol and Ethanol were ordered from VWR. Deuterated DMSO and $\mathrm{CHCl}_{3}$ were purchased from Eurisotop. Und-6DA (methyl undecenoate coupled with hexamethylenediamine) was kindly provided by ITERG company.

\section{Methods and Instruments}

NMR. ${ }^{1} \mathrm{H}$ and ${ }^{13} \mathrm{C}-\mathrm{NMR}$ spectra were recorded on a Bruker Advance 400 spectrometer $(400.20 \mathrm{MHz}$ or $400.33 \mathrm{MHz}$ and $100.63 \mathrm{MHz}$ for $1 \mathrm{H}$ and ${ }_{13} \mathrm{C}$, respectively) using DMSO-d6 or $\mathrm{CDCl}_{3}$ as a solvent at room temperature. The polymerization extent was determined by monitoring the carbonate functions conversion according to NMR spectra, following the disappearance of signals belonging to the carbonate. The peak corresponding to the protons in alpha position of the urethane bond was used as internal reference. No internal or external standard were used for NMR analysis.

FTIR. Infrared spectra were obtained on a Bruker-Tensor 27 spectrometer, equipped with a diamond crystal, using the attenuated total reflection mode. The spectra were acquired from 400 to $4000 \mathrm{~cm}-1$ using 64 scans at a resolution of 4 wavenumbers.
SEC. Polymer molar masses were determined by Size Exclusion Chromatography (SEC) using dimethylformamid (DMF + lithium bromide $\mathrm{LiBr} 1 \mathrm{~g} / \mathrm{L}$ ) as the eluent. Measurements in DMF were performed on an Ultimate 3000 system from Thermoscientific equipped with diode array detector DAD. The system also includes a multi-angle light scattering detector MALLS and differential refractive index detector dRI from Wyatt Technology. Polymers were separated on two different column systems. The first one was two KD-803 Shodex gel columns with a PolyStyrene Divinylbenzene phase $(300 \times 8 \mathrm{~mm}$, exclusion limit: 50 ooo) at a flow rate of $0.8 \mathrm{~mL} / \mathrm{min}$. The second system was composed of two GF310+510 Asahipack columns containing a Polyvinyl alcohol phase $(7.5 \mathrm{x}$ 300, exclusion limit: 300 ooo). Columns temperature was held at $50^{\circ} \mathrm{C}$. Polystyrene was used as the standard and the calibration method is conventional.

$\boldsymbol{T G A}$. Thermogravimetric analyses (TGAs) were performed on the TGA-Q50o system from TA Instruments at a heating rate of $10{ }^{\circ} \mathrm{C} \mathrm{min}^{-1}$ under a nitrogen atmosphere from room temperature to $600^{\circ} \mathrm{C}$, and then under air atmosphere from $600^{\circ} \mathrm{C}$ to $700^{\circ} \mathrm{C}$.

DSC. Differential scanning calorimetry (DSC) thermograms were measured using a DSC Q10o apparatus from TA Instruments. For each sample, two cycles were performed, the first from -40 to $180^{\circ} \mathrm{C}$ at $10^{\circ} \mathrm{C} \mathrm{min}-1$ and a second from $-40^{\circ} \mathrm{C}$ to $180^{\circ} \mathrm{C}$. The glass transition and melting temperatures were calculated from the second heating run.

COMPOUNDER, (twin screw extruder). The reactive compounding was performed using a Micro 5/14 mm twin-screw compounder from thermos Scientific, in a temperature range between 80 and $100{ }^{\circ} \mathrm{C}$ for DGDC and Seb-bCC-ester-based PHUs, and between $120^{\circ} \mathrm{C}$ and $230^{\circ} \mathrm{C}$ for Und-6DA-based PHUs. The rotation speed of the twin screw was kept constantly at $100 \mathrm{rpm}$. The gained $\Delta \mathrm{P}$ - time diagrams were recorded by using Polysoft OS and monitor as software.

\section{5-membered Cyclic Carbonates synthesis}

Diglycerol dicarbonate (DGDC) synthesis: DGDC was synthesized according to previously reported synthesis 36,38 and especially adapted from the work of Bossion et al. $4^{\circ}$ Diglycerol dicarbonate was prepared from diglycerol with a $40 \%$ yield through a one-step procedure. In a round bottom flask, $76 \mathrm{~g}$ (1eq) of diglycerol were introduced and dried under vacuum during $2 \mathrm{~h}$ at $90^{\circ} \mathrm{C}$. Then nitrogen was flushed into the vessel and $1.24 \mathrm{~g}$ (o.05eq) of sodium methoxide catalyst were added under stirring. A condenser was set up above the flask and 5eq $(231 \mathrm{ml})$ of the second reactant, dimethylcarbonate (DMC) were added. The DMC also plays the role of the reaction solvent. The temperature is then set at $110^{\circ} \mathrm{C}$. After $48 \mathrm{~h}$ reaction, the mixture was filtered to remove the sodium methoxide and concentrated by evaporation before two successive recrystallizations in methanol at $-80^{\circ} \mathrm{C}$. DGDC was recovered by filtration on paper filters and dried under vacuum at room temperature. ${ }^{1} \mathrm{H}$ NMR (DMSO-d6, $\left.25^{\circ} \mathrm{C}, 400 \mathrm{MHz}\right), \delta(\mathrm{ppm}): 4.93(\mathrm{~m}$, $\left.{ }_{2} \mathrm{H}, \mathrm{H}_{2}\right), 4.52$ and $4.23\left(\mathrm{t}, 4 \mathrm{H}, \mathrm{H}_{1}\right), 3.74\left(\mathrm{t},{ }_{4} \mathrm{H}, \mathrm{H}_{3}\right) .{ }^{13} \mathrm{C}$ NMR (DMSO-d6, $\left.25^{\circ} \mathrm{C}, 100 \mathrm{MHz}\right), \delta$ (ppm): 154.9 (OCOOO), 74.8 $\left(\underline{\mathrm{CH}}-\mathrm{CH} 2-\mathrm{O}-\mathrm{CH}_{2}\right), 69.8$ ( $\left.\mathrm{CH}-\underline{\mathrm{CH}} 2-\mathrm{O}-\mathrm{CH}_{2}\right), 66.1$ ( $\underline{\mathrm{CH}} 2-\mathrm{CH}-$ $\mathrm{CH} 2-\mathrm{O}-\mathrm{CH} 2)$. IR $\left(\mathrm{cm}^{-1}\right)$ : 2925, 1773, 1476, 1401, 1165. $\mathrm{T}_{\mathrm{m}}=69{ }^{\circ} \mathrm{C}$. Detailed ${ }^{1} \mathrm{H}$ NMR spectrum is shown in Figure SI-1. 
Seb-bCC-ester synthesis. This synthesis was adapted from the work of Carré et al. ${ }^{11}$ In a round-bottom flask, 2.6 eq. (15g) of glycerol carbonate and 4 eq. $(27.24 \mathrm{~mL})$ of triethylamine were diluted in $29.1 \mathrm{~mL}$ of dichloromethane. 1 eq. (11.68 g, 16.7 $\mathrm{mmol}$ ) of sebacoyl chloride was slowly added under an inert atmosphere at $\mathrm{o}{ }^{\circ} \mathrm{C}$. The mixture reaction was left for $2 \mathrm{~h}$ at room temperature. Afterward, the mixture was diluted in 150 $\mathrm{ml}$ of dichloromethane and triethylammonium salts, formed during the reaction, were filtered. The filtrate was then washed three times with $3^{*} 100 \mathrm{~mL}$ of a $10 \% \mathrm{~mol}$. $\mathrm{HCl}$ solution, then three times with $3^{*} 100 \mathrm{~mL}$ of saturated $\mathrm{NaHCO}_{3}$ solution and lastly with $3^{*} 100 \mathrm{~mL}$ of brine. The organic phase was finally dried over anhydrous $\mathrm{MgSO}_{4}$. The Seb-bCC-ester powder was recovered by evaporation and dried under vacuum. Seb-bCC-ester was obtained in $70 \%$ yield as a white powder after evaporation. ${ }^{1} \mathrm{H}$ NMR (DMSO, $25^{\circ} \mathrm{C}$, $\left.400 \mathrm{MHz}\right) \delta(\mathrm{ppm})$ : $5.01(\mathrm{~m}, 2 \mathrm{H}, \mathrm{H} 2), 4.56$ and $4.25(\mathrm{t}, 4 \mathrm{H}, \mathrm{H} 1), 4.29\left(\mathrm{t}, 4 \mathrm{H}, \mathrm{H}_{3}\right), 2.31$ $\left(\mathrm{t},{ }_{4} \mathrm{H}, \mathrm{H}_{4}\right), 1.53\left(\mathrm{~m}, 4 \mathrm{H}, \mathrm{H}_{5}\right), 1.25(8 \mathrm{H}) .{ }^{13} \mathrm{C}$ NMR (DMSO, 25 ${ }^{\circ} \mathrm{C}$, $\left.100 \mathrm{MHz}\right) \delta(\mathrm{ppm}): 173.8$ (CH2-OCOO-CH2), 154.1 (OCOOO), 74.3 ( $\left.\mathrm{CH}-\mathrm{CH}_{2}-\mathrm{OCO}\right), 66.4$ ( $\left.\underline{\mathrm{CH}} 2-\mathrm{CH}-\mathrm{CH}_{2}-\mathrm{OCO}\right), 62.8$ ( $\mathrm{CH}-$ CH2-OCO), 33.3 (OCO- $\mathrm{CH}_{2}-\mathrm{CH}_{2}$ ), 28.5-28.2 ( $\left.\mathrm{CH}_{2}\right), 24.2$ (OCO-CH2- $\left.\mathrm{CH}_{2}\right)$. IR ( $\left.\mathrm{cm}^{-1}\right): 2936,2845,1778,1730 . \mathrm{T}_{\mathrm{m}}=84$ ${ }^{\circ} \mathrm{C}$. Detailed ${ }^{1} \mathrm{H}$ NMR spectrum is shown in Figure SI-11.

Und-6DA-bisCC. 1. Epoxidation reaction. The initial Und-6DA (methyl undecenoate coupled with hexamethylenediamine) (5og, 1eq) and m-CPBA (50,7g, 3 eq) were stirred at room temperature in chloroform. After 1 day, the conversion of the double bonds, monitored by ${ }^{1} \mathrm{H}$ NMR spectroscopy (see figure SI22), was complete. The purification of the product was done in two steps. First, the organic layer was washed over aqueous $\mathrm{Na}_{2} \mathrm{SO}_{3}(3 \times 500 \mathrm{~mL})$, aqueous $\mathrm{NaHCO}_{3}(4 \times 500 \mathrm{~mL})$, neutralized with water and dried over anhydrous sodium sulfate. Solvent was then removed after filtration. Secondly, the bis-epoxide was dispersed in ethanol and centrifuged. After the removal of the supernatant, the precipitated bis-epoxide was then recovered and dried under vacuum. This procedure allows the correct removal of m-CPBA. 2. Carbonation reaction. The bis-epoxide was first pre-mixed with $\mathrm{TBABr}$ ( $3 \mathrm{wt} \%)$. Then the mixture was placed in a reactor and $\mathrm{CO}_{2}$ was slowly introduced into the reactor until 50 Bars. The vessels was heated up at $140^{\circ} \mathrm{C}$ and let under magnetic stirring. After 24 hours, the reactor was cooled down to room temperature and slowly depressurized to the atmospheric pressure. All the ${ }^{1} \mathrm{H}$ NMR of all products revealed quantitative conversion by the disappearance of the protons of the epoxide. Yield: $95 \%$. ${ }^{1} \mathrm{H}$ NMR $\left(\mathrm{CDCl}_{3,25}{ }^{\circ} \mathrm{C}, 400 \mathrm{MHz}\right) \mathrm{d}(\mathrm{ppm}): 5.83(\mathrm{~s}, 2 \mathrm{NH}), 4.70(\mathrm{~m}, 2 \mathrm{H})$, $4.53(\mathrm{t}, 2 \mathrm{H}), 4.06(\mathrm{t}, 2 \mathrm{H}), 3.26(\mathrm{~m}, 4 \mathrm{H}), 2.16(\mathrm{t}, 4 \mathrm{H}), 1.78(\mathrm{~m}$, $2 \mathrm{H}), 1.62(\mathrm{~m}, 6 \mathrm{H}), 1.53(\mathrm{~m}, 4 \mathrm{H}), 1.47(\mathrm{~m}, 4 \mathrm{H}), 1.30(\mathrm{~m}, 16 \mathrm{H}) .13 \mathrm{C}$ NMR $\left(\mathrm{CDCl}_{3}, 25^{\circ} \mathrm{C}\right.$, $\left.100 \mathrm{MHz}\right) \mathrm{d}(\mathrm{ppm}): 173.81$ (CONH), 155.27 (OCOO), 77.22 (CH-OCOO), 69.54 ( $\left.\mathrm{CH}_{2}-\mathrm{OCOO}\right), 39.20$ $\left(\mathrm{CH}_{2}-\mathrm{NHCO}\right), 36.65 \quad\left(\mathrm{CH}_{2}-\mathrm{CONH}\right), 33.94 \quad\left(\mathrm{CH}_{2}-\mathrm{CH}-\right.$ OCOO), $29.17\left(\mathrm{CH}_{2}\right), 26.91\left(\mathrm{CH}_{2}-\mathrm{CH}_{2}-\mathrm{NHCO}\right), 25.84\left(\mathrm{CH}_{2}-\right.$ $\left.\mathrm{CH}_{2}-\mathrm{CONH}\right), 24.43$ ( $\left.\mathrm{CH}_{2}-\mathrm{CH}_{2}-\mathrm{CH}-\mathrm{OCOO}\right) . \mathrm{IR}\left(\mathrm{cm}^{-1}\right)$ : 3309, 2918, 2850, 1778, 1637, 1535. $\mathrm{T}_{\mathrm{m}}=114^{\circ} \mathrm{C}$. Detailed ${ }^{1} \mathrm{H}$ NMR spectrum of Und-6DA-bisCC can be seen in Figure SI-23. Procedure summary available in Figure SI-21.

Standard procedure for polymerization by reactive extrusion process. Bis-CCs and diamines monomers, generally both powders, were weighed in a stoichiometric ratio of 1 and manually premixed for one minute. Then, the mixture was slowly injected in the micro compounder, without initial pressure. The solvent-free polymerizations were performed from $80^{\circ} \mathrm{C}$ to $95^{\circ} \mathrm{C}$, depending on the diamine nature. The reactants injection usually lasts 5 minutes and was done manually, without pushing the monomers to avoid losses. The twin-screw rotation speed was set at $100 \mathrm{rpm}$ and kept steady during the whole reaction. Torque, actual temperature and pressure measured inside the reactor were followed by PolySoft monitor software. When a liquid diamine was used, bisCCs were first injected into the hot reactor in order to be melted, and then the appropriate volume of the diamine was added with a syringe. In the specific case of Und-6DA-bisCC polymerizations, the temperature was started $120^{\circ} \mathrm{C}$ with $10 \mathrm{DA}, 6 \mathrm{DA}$ and $\mathrm{m}$-xylylene DA and at $220^{\circ} \mathrm{C}$ in the case of L-Lysine. In order to ensure the good circulation of the materials during the polymerization by reactive extrusion of those amide-type carbonates, a pressure dependent temperature program was created, in order to level the pressure in the channel. If the pressure recorded on pressure sensor number 1 (p-Di) overcome 100 bars, the temperature was increased by $5^{\circ} \mathrm{C}$. The temperature increases allowed to reduce the viscosity of the polymer material, reducing the global pressure in reactor. The pressure in the channel, labelled $\Delta \mathrm{P}$ is actually the difference between two pressures sensors located at each edge of the circulation channel. For $\mathrm{PHU}_{15}$ and $\mathrm{PHU}_{17}$, the pressure on $\mathrm{p}$-D1 never overcame 100 bars and, as a consequence, temperature was kept constant. For $\mathrm{PHU}_{16}$, temperature had to be adjusted. For $\mathrm{PHU}_{18}$, the temperature was increased, but considering the fact that those increases did not have much impact on the pressure (very dense material created), and due to the already high temperature values, $230^{\circ} \mathrm{C}$ was put as an upper limit.

\section{Standard procedure for polymerization with magnetic or} mechanical stirring.

Bis-CCs (1 eq, 2g) were introduced in schlenk tubes and heated at the desire temparture (oil bath). Then the stirring tool, either magnetic stirrer or the blade for mechanical stirring is added in the schlenck. For mechanical stirring the stirring blade is screwed to a IKA RW20 Digital instrument. The bisCCs are melted and stirred during $5 \mathrm{~min}$ and the appropriate amount of diamine is added ( $1 \mathrm{eq}$ ) under stirring. This method allow the immediate mixing of the diamine with the melted bisCC, preventing its sublimation. After the apropriate reaction time, the stirring is stopped, the schlenk tube is immediately cooled down at room temperature and samples analyzed.

\section{RESULTS AND DISCUSSION}

\section{DGDC-based PHUs synthesis through reactive extrusion}

Polyhydroxyurethanes were first synthesized from DGDC and two aliphatic diamines, respectively hexamethylene diamine $\left(6 \mathrm{DA}, \mathrm{PHU}_{1}\right)$ and 1.10 diaminodecane (10DA, $\mathrm{PHU}_{2}$ ), as displayed in Figure 1.

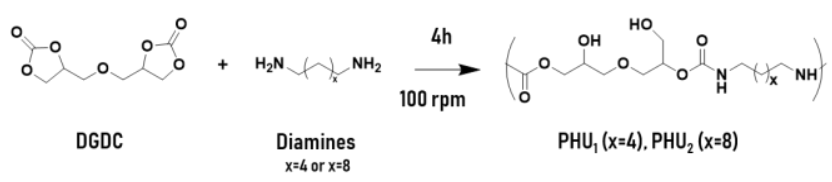

Figure 1. Synthesis of DGDC-based PHUs with aliphatic diamines $(x=4$ for $6 D A$ or $x=8$ for 1oDA) 
In both cases, the polymers were extruded after $4 \mathrm{~h}$ and cooled down at room temperature. The polymerization rate was followed by monitoring the pressure drop $(\Delta \mathrm{P})$, recorded over time by two pressure sensors located at each end of the circulation channel.

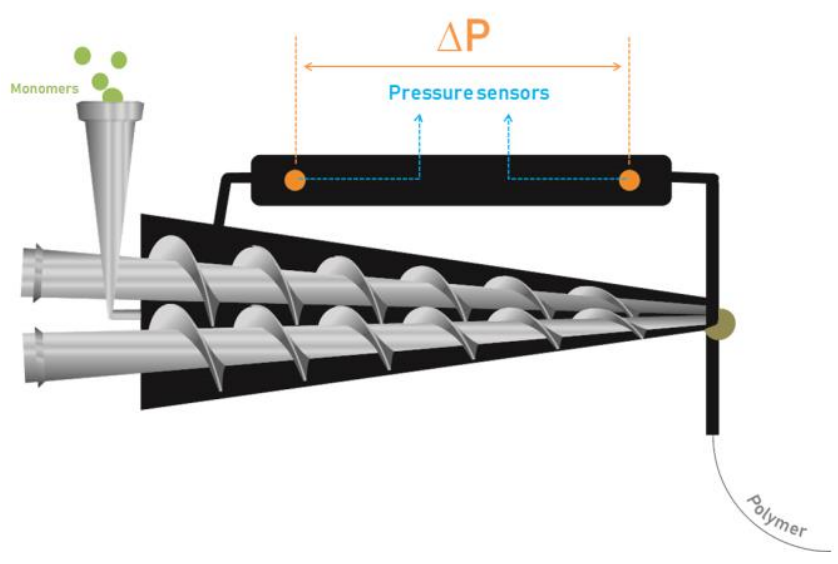

Figure 2. Scheme of the extruder reactor (Inside view). Orange circles represent the two pressure sensors.

The scheme presented in Figure 2 shows in black the hollow part of the extruder where the polymer is formed and circulates during the polymerization. The two orange circles at each edge of the circulation channel represent the two pressure sensors. As clearly understandable from the scheme, when the polymer reaches the end of the twin screws, it is reinjected at the entrance of the screws through the circulation channel. Several parameters such as temperature, screws rotation speed and obviously residence time can be tuned, thus affecting the chemistry itself but also the rheology within the extruder. In this sense, the final properties and mainly the homogeneity of the PHUs so-formed deeply depend on these parameters.

Benyahya et. al $4^{1}$ have shown that optimal temperature for PHU synthesis is around $80^{\circ} \mathrm{C}$ and should not be over $120^{\circ} \mathrm{C}$. Indeed, several works ${ }^{42,43}$ demonstrated that the formation of urea moieties, the major by-product in poly(hydroxyurethane)s synthesis, occurs when the temperature gets close to $100^{\circ} \mathrm{C}$ and above. Polymerization temperatures were chosen according to those observations, added to the fact that bulk polymerizations require temperature higher than the melting point of monomers. In the case of DGDC polymerization with $6 \mathrm{DA}$, the reaction temperature was kept at $80^{\circ} \mathrm{C}$. As semicrystalline PHUs were obtained with DGDC / 10DA polymerization, the reactive extrusion process was adapted to ensure a good homogeneity of the blend. The time vs temperature program was as follows: start at $80^{\circ} \mathrm{C} / 45 \mathrm{~min}$, then $85^{\circ} \mathrm{C} / 15 \mathrm{~min}$, $90^{\circ} \mathrm{C} / 15 \mathrm{~min}$ and finally $95^{\circ} \mathrm{C} / 165 \mathrm{~min}$ (see Figure 3 ) to guarantee the melting of the entire polymer chains. In this specific case, the reaction was started at the lowest temperature possible for three main reasons: lowering the formation of urea moieties, ensure the efficient circulation of the polymer in the channel and preventing the sublimation of rather low vapour pressure 10DA. Indeed, a higher starting temperature would lead to a partial loss of diamine through sublimation, compromising stoichiometry compliance. Besides, when the substance within the reactor is too liquid (which is the case when the reaction is started at $95^{\circ} \mathrm{C}$ ), the pressure induced by the screws is less efficient, compromising the correct circulation of the polymer within the extruder. Figure 3 shows $\Delta \mathrm{P}$ recorded over time during $\mathrm{PHU}_{1}$ synthesis at constant temperature (top) and the $\Delta \mathrm{P}$ profile obtained during $\mathrm{PHU}_{2}$ synthesis (bottom). In the case of $\mathrm{PHU}_{1}$, the plateau reached after 4 hours of reaction shows that the viscosity of the system was no longer increasing, behavior attributed to the completion of the reaction. For $\mathrm{PHU}_{2}$, even if the $\Delta \mathrm{P}$ was still increasing during the last two hours, it was decided to stop the polymerization after 4 hours, in order to keep short reaction times. As can be observed for $\mathrm{PHU}_{2}$, the pressure, and so the viscosity, drastically decreased when the temperature was increased from $80^{\circ} \mathrm{C}$ to $95^{\circ} \mathrm{C}$. This behaviour is obviously characteristic of the thermoplastic feature of the obtained PHUs.

After extrusion, $\mathrm{PHU}_{1}$ and $\mathrm{PHU}_{2}$ were analysed by proton $\mathrm{NMR}$, in order to quantify the conversion of reactive functions. In both cases, the carbonate conversion was over $99 \%$, as shown by the stacked ${ }^{1} \mathrm{H}$ NMR spectra displayed in Figure 4. In order to confirm the high conversion, additional ${ }^{1} \mathrm{H}$ NMR spectra were recorded with 1024 scans (see Figure SI-2 and Figure SI-3) and FTIR spectroscopy was performed (see Figure SI4). Carbonate conversion was calculated by integrating the remaining peak of carbonate ring between 4.2 and 4.3 ppm.
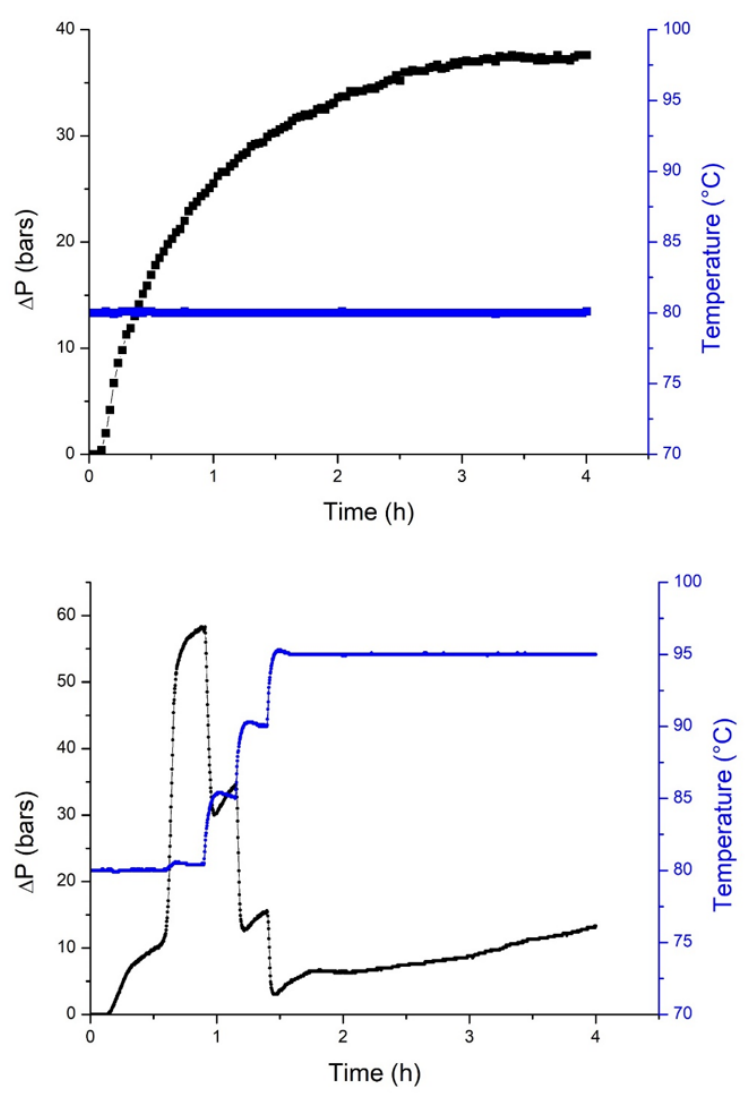

Figure 3. Pressure loss curve over time recorded during polymerization by reactive extrusion of: DGDC and 6DA $\left(\mathrm{PHU}_{1}\right.$, top), DGDC and 1oDA (PHU, bottom $)$ 
1

DGDC
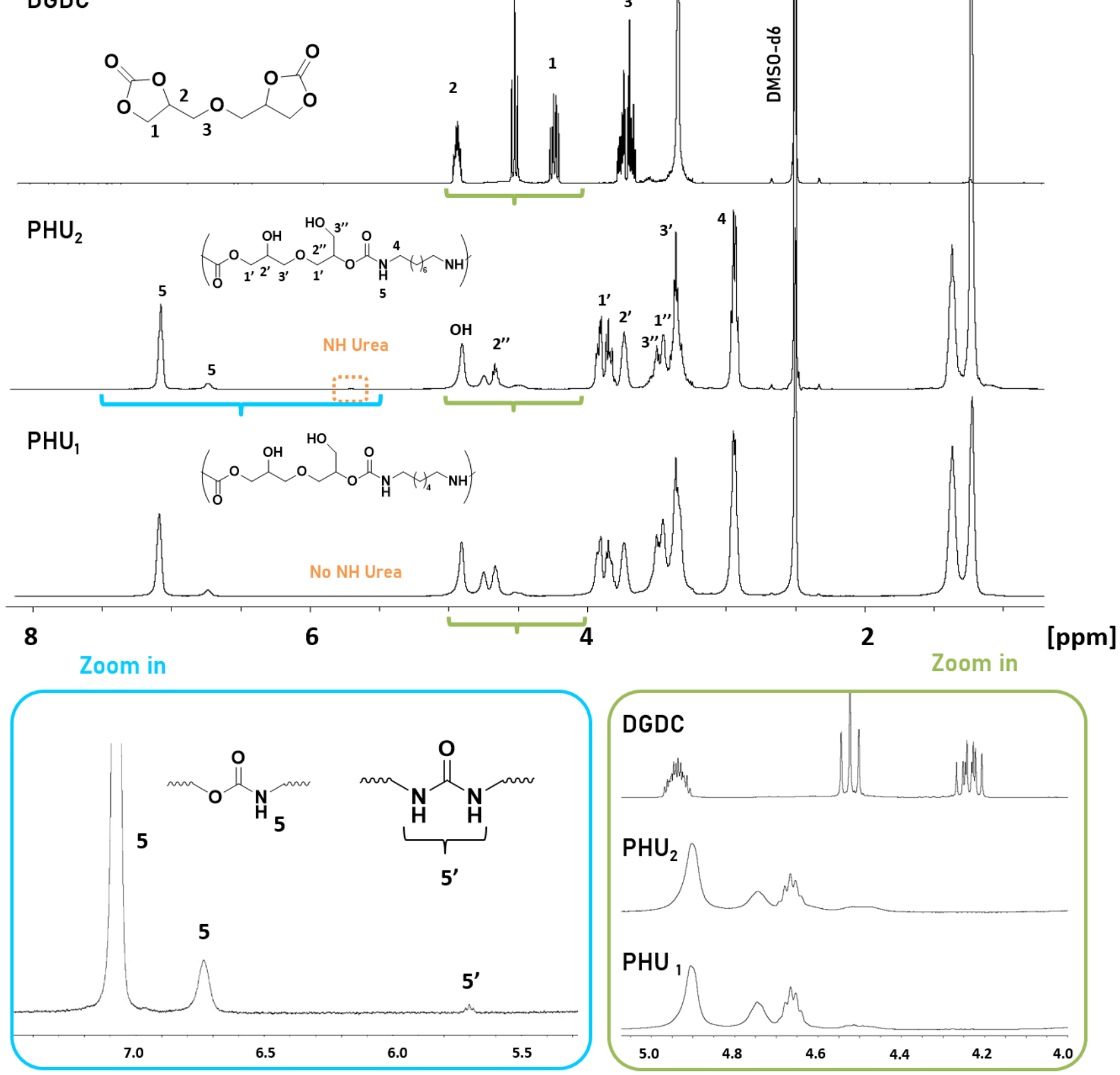

Figure 4. Stacked ${ }^{1} \mathrm{H}$ NMR spectra in DMSO-d6 of DGDC monomer, $\mathrm{PHU}_{1}$ and $\mathrm{PHU}_{2}$ after 4 hours of reactive extrusion polymerization. Bottom Left: Zoom on DGDC remaining zone 4.0-5.0 ppm. Bottom Right: Zoom on urea and urethane peaks 5.4-7.4 $\mathrm{ppm}$.

Besides, ${ }^{1} \mathrm{H}$ NMR spectra show that no urea was formed when 6DA was polymerized with DGDC at $80^{\circ} \mathrm{C}$, whereas the appearance of a small peak (1.1\%) at $5.7 \mathrm{ppm}$ can be noticed on $\mathrm{PHU}_{2}$, in the case of DGDC / 10DA polymerization.

This peak can be undoubtedly attributed to urea functions according to previous studies 40,43. A zoom of this spectrum zone is displayed in Figure 4.

Finally, the thermal properties of the PHUs were determined by DSC and TGA analysis. $\mathrm{PHU}_{1}$ synthesized from DGDC and 6DA was obtained as an amorphous material. In the case of
10DA, a semi crystalline polymer was obtained for $\mathrm{PHU}_{2}$, with crystallization and melting temperature respectively at $94.6^{\circ} \mathrm{C}$ and $103^{\circ} \mathrm{C}$. This temperature found for crystallization confirms the necessity to increase the temperature up to $95^{\circ} \mathrm{C}$ during polymerization (temperature program previously described) to overcome the crystallisation of the so-created PHU in this extruder. Besides, TGA traces showed thermal stabilities until $204{ }^{\circ} \mathrm{C}$ and $216^{\circ} \mathrm{C}$ for $\mathrm{PHU}_{1}$ and $\mathrm{PHU}_{2}$ respectively, according to the $\mathrm{T}_{\mathrm{d} 5 \%}$ measurement. The second heating cycle of DSC analysis displayed $\mathrm{T}_{\mathrm{g} S}$ of $16^{\circ} \mathrm{C}$ and $5^{\circ} \mathrm{C}$ respectively. 
Comparison with bulk PHU synthesis using magnetic and mechanical stirring.

In order to evaluate the real benefit brought by the reactive extrusion process on the PHU synthesis, comparative bulkpolymerizations were performed using magnetic or mechanical stirrings. As shown in Table $1, \mathrm{PHU}_{3}$ and $\mathrm{PHU}_{4}$ have beensynthesized from DGDC and 6DA or 10DA respectively, in a Schlenk tube under magnetic stirring.

The polymerization temperatures were the same as previously tested by reactive extrusion, meaning $80^{\circ} \mathrm{C}$ with $6 \mathrm{DA}$ and the established temperature ramp (from $80^{\circ} \mathrm{C}$ to $95^{\circ} \mathrm{C}$ ) for $\mathrm{PHU}_{4}$. The polymers were recovered after $4 \mathrm{~h}$ of polymerization and analyzed by ${ }^{1} \mathrm{H}$ NMR and SEC. $\mathrm{PHU}_{3}$ and $\mathrm{PHU}_{4}$ showed a conversion of carbonate functions of only $48.72 \%$ and $40.9 \%$ respectively, demonstrating the extremely poor efficiency of magnetic stirring for PHU bulk polymerizations. Detailed 'HNMR spectra are displayed in Figures SI-5. Simultaneously, a series of bulk polymerizations were then performed under mechanical stirring, following the conditions already described (see Table $1, \mathrm{PHU}_{5}$ and $\mathrm{PHU}_{6}$ ). In both last cases, the carbonate conversions were found very high after $4 \mathrm{~h}$ reaction time, comparable to the results obtained by reactive extrusion ( ${ }^{\mathrm{H}}$ NMR displayed in Figure SI-6). For comparison, SEC traces in $\mathrm{DMF}$ of $\mathrm{PHU}_{1}, \mathrm{PHU}_{3}$ and $\mathrm{PHU}_{5}$ are displayed in Figure 5. Same comparison with $\mathrm{PHU}_{2}, \mathrm{PHU}_{4}$ and $\mathrm{PHU}_{6}$ made from 1oDA, is available in Figure SI-7.

The red SEC trace corresponding to $\mathrm{PHU}_{3}$ prepared at $80^{\circ} \mathrm{C}$ with magnetic stirring, shows the presence of oligomers in significant amount. With mechanical stirring $\left(\mathrm{PHU}_{5}\right.$, black curve), the amount of oligomers is still detectable but drastically decreased. In agreement with NMR analysis, the polymerization performed by reactive extrusion (blue curve), at $80^{\circ} \mathrm{C}$ after 4 h reaction time, enables the apparent total consumption of carbonate functions, leading to minimum quantities of visible oligomers, and the formation of higher mola masses. All these data confirm that an efficient mixing is essential to ensure the monomer diffusion during the polymerization.

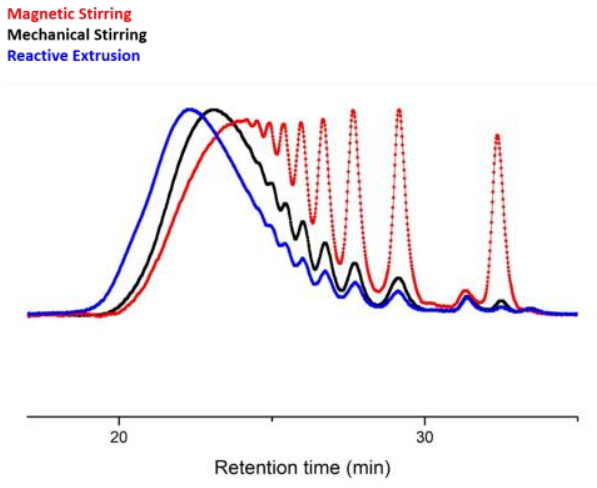

Figure 5. SEC traces in DMF Asahipack set of columns of $\mathrm{PHU}_{3}, \mathrm{PHU}_{5}$ and $\mathrm{PHU}_{1}$, all synthesized with DGDC and 6DA at $80^{\circ} \mathrm{C}$, through magnetic stirring (red), mechanical stirring (black) and reactive extrusion (blue)

As could be expected, these results demonstrated that keeping the homogeneity of the reactive medium is a way to achieve a quasi complete conversion of the reactive functions and consequently to obtain the highest molar masses. To this purpose, the mechanical stirring gives relatively good results but the reactive extrusion process provides the highest molar masses thanks to a better mixing notably in the case of high viscous systems. In addition, a second main advantage of the extrusion process relates to the process by itself which enables the manufacture of pieces, as discussed further in the manuscript.

\section{PHU molar mass determination}

PHUs molar masses were determined using size exclusion chromatography in DMF, with two different systems of columns. In both cases, molecular weights were calculated with the universal method, related to polystyrene standards. The first series of columns were filled with a gel of PS (Shodex). With this chromatographic system, the molar masses of $\mathrm{PHU}_{1}$ and $\mathrm{PHU}_{2}$ were respectively equal to $37400 \mathrm{~g} / \mathrm{mol}$ and 35600 $\mathrm{g} / \mathrm{mol}$ with dispersities values around 1.2 to 1.3 . Such dispersity values are not in agreement with high conversion values measured by NMR (Figure 4 and Table 1). Considering this observation, PHUs were also analyzed with a second set of columns filled with a polyvinyl alcohol gel (Asahipack). In this case, the molar masses obtained for $\mathrm{PHU}_{1}$ and $\mathrm{PHU}_{2}$ were equal to $5000 \mathrm{~g} / \mathrm{mol}$ with $Đ=\mathbf{2 . 5}$ and $5600 \mathrm{~g} / \mathrm{mol}$ with $Đ=1.96$, respectively. In this latter case, dispersity values are more consistent with Carothers theory and in agreement with the complete carbonate conversion. However, the relative molar masses appear, in this case, underestimated. Indeed, considering these values, the calculated polymerization degrees would be around 16 both for $\mathrm{PHU}_{1}$ and $\mathrm{PHU}_{2}$. These size exclusion chromatography analyses confirm how it is difficult to estimate the relative molar masses of polyhydroxyurethanes based on polystyrene calibrations, as already discussed in literature data. 44 However, despite this issue, one could compare the SEC data of the prepared PHUs with respect to the process implemented, reactive extrusion vs conventional bulk polymerization.

Versatility of the reactive extrusion process for PHU synthesis.

In order to further investigate the potential of the reactive extrusion process to produce PHU materials, another type of activated bisCC, named Seb-bCC-ester, was tested. Seb-bCC-ester was synthesized in one step from sebacoyl-chloride and glycerol carbonate ${ }^{11}$. To ensure the good melting of the SebbCC-ester $\left(\mathrm{T}_{\mathrm{m}}=84^{\circ} \mathrm{C}\right)$, the polymerization temperature was set at $90^{\circ} \mathrm{C}$ and the twin screws rotation speed fixed at 100 rpm. Thanks to the ester bond in beta position of the carbonate ring, the reactivity of Seb-bCC-ester towards aminolysis is high, compared to that of DGDC. However, one issue with this monomer is the possibility to form amide linkages within the PHU backbone. As already described, this secondary reaction between ester and amine reactive groups can occur till $70^{\circ} \mathrm{C} .{ }^{13}$ Due to these particularities, the protocol for reactive extrusion polymerization involving Seb-bCC-ester as bis-carbonate monomer was slightly different than the one for DGDC-based PHUs. Indeed, the PHUs so-formed quickly became highly viscous materials, leading to a drastic increase in terms of torque and pressure $(\Delta \mathrm{P})$ within the channel. 
Table 1: DGDC-based PHUs, synthesized in bulk with Magnetic Stirring, Mechanical Stirring or through Reactive Extrusion process

\begin{tabular}{|c|c|c|c|c|c|c|c|c|c|c|}
\hline & \multirow{2}{*}{$\begin{array}{l}\mathbf{P} \\
\mathbf{H} \\
\mathbf{U}\end{array}$} & \multirow{2}{*}{ DA } & \multirow{2}{*}{$\begin{array}{c}\text { Temperature } \\
\left({ }^{\circ} \mathrm{C}\right)\end{array}$} & \multirow{2}{*}{ Duration } & \multirow{2}{*}{$\begin{array}{c}C C \text { con- } \\
\text { version } \\
(\%)^{a}\end{array}$} & \multirow{2}{*}{$\begin{array}{c}\text { Ratio Urea / } \\
\text { Urethane }^{\mathrm{a}}\end{array}$} & \multicolumn{2}{|c|}{$\mathbf{M}_{\mathrm{n}}[Đ](\mathrm{g} / \mathrm{mol})$} & \multirow{2}{*}{$\begin{array}{c}\mathrm{T}_{\mathrm{g}} \\
\left({ }^{\circ} \mathbf{C}\right) \\
\mathrm{d}\end{array}$} & \multirow{2}{*}{$\begin{array}{l}\mathrm{T}_{\mathrm{d} 5 \%} \% \\
\left({ }^{\circ} \mathbf{C}\right) \\
\text { e }\end{array}$} \\
\hline & & & & & & & Shodex ${ }^{b}$ & $\begin{array}{l}\text { Asahi- } \\
\text { pack }^{\mathrm{c}}\end{array}$ & & \\
\hline \multirow{2}{*}{$\begin{array}{l}\text { MAGNETIC } \\
\text { STIRRING }\end{array}$} & 3 & $6 \mathrm{DA}$ & 80 & $4 \mathrm{~h}$ & 49 & $0 / 100$ & $16100[1.5]$ & $2100[2.6]$ & 8 & $\mathrm{Nd}$ \\
\hline & 4 & $10 \mathrm{DA}$ & $\mathrm{RP}$ & $4 \mathrm{~h}$ & 41 & $0 / 100$ & $16300[1.3]$ & $1700[1.9]$ & 5 & 189 \\
\hline \multirow{2}{*}{$\begin{array}{l}\text { MECHANICAL } \\
\text { STIRRING }\end{array}$} & 5 & $6 \mathrm{DA}$ & 80 & $4 \mathrm{~h}$ & $>99$ & $0 / 100$ & $22800[1.3]$ & $3700[2.3]$ & 19 & 207 \\
\hline & 6 & $10 \mathrm{DA}$ & $\mathrm{RP}$ & $4 \mathrm{~h}$ & $>99$ & $0.3 / 99.7$ & $17100[1.4]$ & $3100[2.0]$ & 5 & 209 \\
\hline \multirow{2}{*}{$\begin{array}{c}\text { REACTIVE } \\
\text { EXTRUSION }\end{array}$} & 1 & $6 \mathrm{DA}$ & 80 & $4 \mathrm{~h}$ & $>99$ & o / 100 & 34400 [1.4] & $5000[2.5]$ & 16 & 206 \\
\hline & 2 & 10DA & $\mathrm{RP}$ & $4 \mathrm{~h}$ & $>99$ & $1.1 / 98.9$ & $35600[1.3]$ & $5600[1.9]$ & 5 & 219 \\
\hline
\end{tabular}

a: Obtained by ${ }^{1} \mathrm{H}_{\mathrm{NMR}}{ }^{\mathrm{b}}$ : Obtained from SEC analysis in DMF, with LiBr salts, Toluene as flow marker and the KD803 Shodex Columns with PS Divinyl benzene phase. ${ }^{\mathrm{C}}$ : Obtained from SEC analysis in DMF, with LiBr salts, Toluene as flow marker and the Asahipack Columns with Polyvinyl Alcohol phase. ${ }^{\mathrm{d}}$ : DSC second heating cycle. e: TGA, RT to $600^{\circ} \mathrm{C}$ under $\mathrm{N} 2$ flow. RP: Ramp Protocol: $80^{\circ} \mathrm{C} / 85^{\circ} \mathrm{C} / 90^{\circ} \mathrm{C} / 95^{\circ} \mathrm{C}$ : $45 \mathrm{~min} / 15 \mathrm{~min} / 15 \mathrm{~min} / 165 \mathrm{~min}$. Nd : Not Determined.

The polymerizations were let until the blocking of the extruder, due to high threshold torque and/or $\Delta \mathrm{P}$ reached. The Seb-bCCester-based PHUs were taken out directly molded into the circulation channel to obtain brown rubbery materials with a bar-like shape (Figure 6). Consequently, the polymerization duration was comprised between 21 minutes and one hour and a half depending on the monomer systems (Seb-bCC-ester/various aliphatic diamines). The temperature was not increased higher than $90^{\circ} \mathrm{C}$ in order to minimize the above mentioned secondary reactions. Different aliphatic diamines were tested as co-monomers with SebbCC-ester; 1.4-diaminobutane (4DA, $\left.\mathrm{PHU}_{9}\right)$, 1.6-diaminohexane (6DA, $\mathrm{PHU}_{10}$ ) 1.10-diaminodecane (1oDA, $\mathrm{PHU} \mathrm{U}_{11}$ ), and 1.12-diaminododecane (12DA, $\mathrm{PHU}_{12}$ ) as detailed in Table 2.<smiles>O=C(CCCCCCCCC(=O)OCC1COC(=O)O1)OCC1COC(=O)O1</smiles>

Seb-bCC-ester
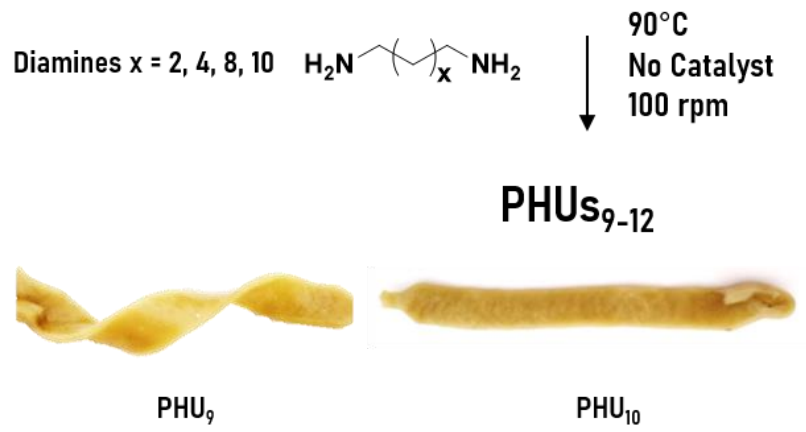

Figure 6. Synthesis of $\mathrm{PHU}_{9-12}$ from Seb-bCC-ester with different diamines, and pictures of PHUs directly taken out from the extruder.

As these PHUs were insoluble in most classical organic solvents, such as DMSO or DMF for instance, their characterization by NMR and SEC was not easily feasible. However, FTIR spectroscopy analyses have been performed. The FTIR spectra presented in Figure 7 show the expected bands corresponding to urethane bond $(v=1685 \mathrm{~cm}-1)$, in which the slightly visible shoulder $(v=1733$ $\mathrm{cm}-1)$ is representative of the ester moieties in beta position of the urethane. 


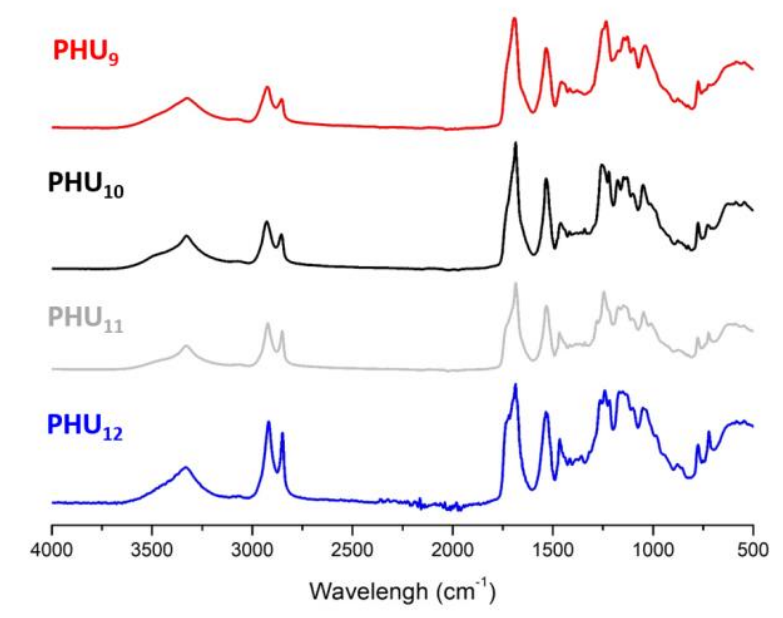

Figure 7. Stacked FTIR spectra of $\mathrm{PHU}_{9}$ (black), $\mathrm{PHU}_{10}$ (blue), $\mathrm{PHU}_{11}$ (red), PHU12 (grey).

The band corresponding to $\mathrm{NH}$ and $\mathrm{OH}$ vibrations $\left(v=3400 \mathrm{~cm}^{-1}\right)$ is also visible. Interestingly, this analysis confirms the complete conversion of carbonate functions, as attested by the absence of remaining band at $v=1782 \mathrm{~cm}^{-1}$, corresponding to the carbonyl of the cyclic carbonate ring. This result confirms the good homogenization of the reactive medium within the extruder until it is blocked. As expected, the extrusion process contributes to shorten the reaction times drastically thus limiting the occurrence of secondary reactions. In addition, and more importantly, the conversion of carbonate functions was complete without requiring any catalysts. Regarding the amount of by-products contained in the so-created PHUs, the ratio amide/urethane/urea was calculated, as example, for $\mathrm{PHU}_{10}$. HR-MAS and ${ }^{~} \mathrm{H}$ NMR analysis were performed respectively on the insoluble and soluble fractions, in DMSO-d6 (see Figure SI-12).

The integration of NMR signals gave the following ratios: 5.4/92.8/1.7 for the insoluble part, and 5.6/94.4/o for the soluble fraction of $\mathrm{PHU}_{10}$. These results demonstrate that the amount of by-products in Seb-bCC-ester based PHUs remains very low, discharging side reactions from the insolubility issues. Besides, $\mathrm{PHU}_{9-12}$ were found to be completely soluble In 1,1,1,3,3,3-Hexafluoro-2-propanol (HFIP), proving the absence of cross-linking in the material. It was finally hypothesized that the insoluble fraction in DMSO might contain high molar masses chains. This feature will be further discussed.

\section{Thermal properties of Seb-bCC-ester based PHUs}

PHUs thermal stabilities $\left(\mathrm{T}_{\mathrm{d} 5 \%}\right)$ and $\mathrm{T}_{\mathrm{g}} \mathrm{s}$ were determined by TGA and DSC, respectively. These values are affected by the diamine chain length: the longer the diamine (from $4 \mathrm{DA}$ to ${ }_{12} \mathrm{DA}$ ), the lower the glass transition temperature. Indeed, a longer diamine chain length leads to an increase of chain mobility, decreasing the energy needed to induce the glass transition temperature phenomenon. This observation was already made by Benyaha et al., demonstrating the relation between diamine structure and final polymer properties.45 In addition, the thermal stability of the PHUs is directly correlated to the thermal stability of the diamine. The longer the aliphatic diamine, the higher the PHU thermal stability (see Figure 8). Seb-bCC-ester based PHUs were then obtained with relatively high thermal stability up to $250^{\circ} \mathrm{C}$, with a range of $\mathrm{T}_{\mathrm{gs}}$ from $-0.9^{\circ} \mathrm{C}$ to $19^{\circ} \mathrm{C}$. TGA and DSC second heating cycle curves can be seen in Figures SI-13 and SI-14.

The above-mentioned results demonstrate that the reactive extrusion process can be efficiently applied to PHUs synthesis and adapted to a wide range of monomers keeping the consistency of the expected final polymer properties.

Table 2: Thermal properties of Seb-bCC-ester based PHUs synthesized at $90^{\circ} \mathrm{C}$ through Reactive Extrusion.

\begin{tabular}{c|ccccc} 
PHU & DA & $\begin{array}{c}\text { Polymeri- } \\
\text { zation } \\
\text { Time }\end{array}$ & $\begin{array}{c}\mathbf{T}_{\mathbf{g}} \\
\left({ }^{\circ} \mathbf{C}\right)\end{array}$ & $\begin{array}{c}\mathbf{T}_{\mathbf{d} 5 \%} \\
\left({ }^{\circ} \mathbf{C}\right)\end{array}$ & $\begin{array}{c}\mathbf{T}_{\mathbf{m}(\mathbf{s})} \\
\left({ }^{\circ} \mathbf{C}\right)\end{array}$ \\
\hline 9 & $4 \mathrm{DA}$ & $1 \mathrm{~h} 26$ & 8.1 & 217 & -- \\
10 & $6 \mathrm{DA}$ & $1 \mathrm{~h}$ & 3.8 & 220 & -- \\
11 & $10 \mathrm{DA}$ & $21 \mathrm{~min}$ & 0 & 239 & $54 / 78$ \\
12 & $12 \mathrm{DA}$ & $1 \mathrm{~h} 5$ & -0.9 & 250 & $58 / 89$
\end{tabular}




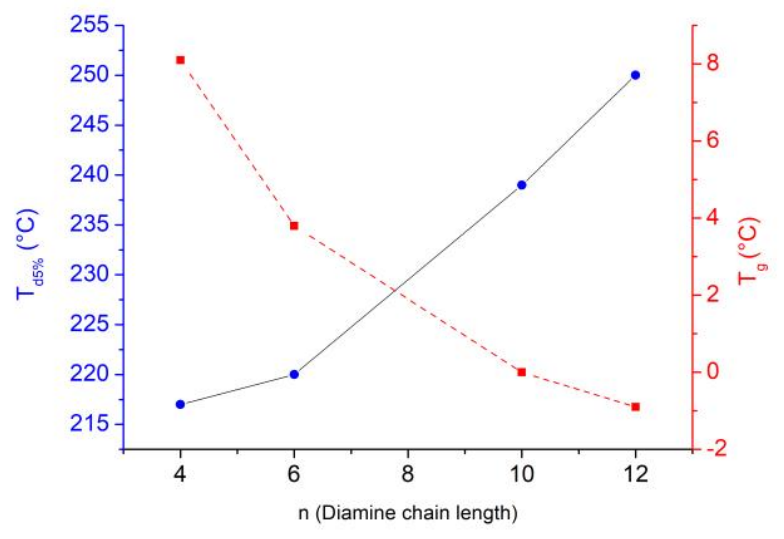

Figure 8. $\mathrm{T}_{\mathrm{g}} \mathrm{s}$ and $\mathrm{T}_{\mathrm{d} 5} \%$ curves of $\mathrm{PHU}_{8}$ to $\mathrm{PHU}_{11}$ as a function of diamines chain length (n).

Finally, in order to confirm the results previously demonstrated on DGDC regarding the efficiency of reactive extrusion compared to classical polymerization, the synthesis of $\mathrm{PHU}_{10}\left(\mathrm{DGDC} / 6 \mathrm{DA} / 90^{\circ} \mathrm{C} / \mathrm{h} \mathrm{h}\right)$ was reproduced, using magnetic and mechanical stirring ( $\mathrm{PHU}_{13}$ and $\mathrm{PHU}_{14}$, see Table 3).

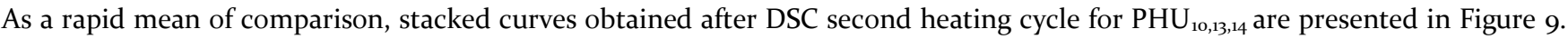
As expected, the highest $\mathrm{T}_{\mathrm{g}}$ was obtained for $\mathrm{PHU}_{10}$, synthesized through extrusion and the second highest was with mechanical stirring.

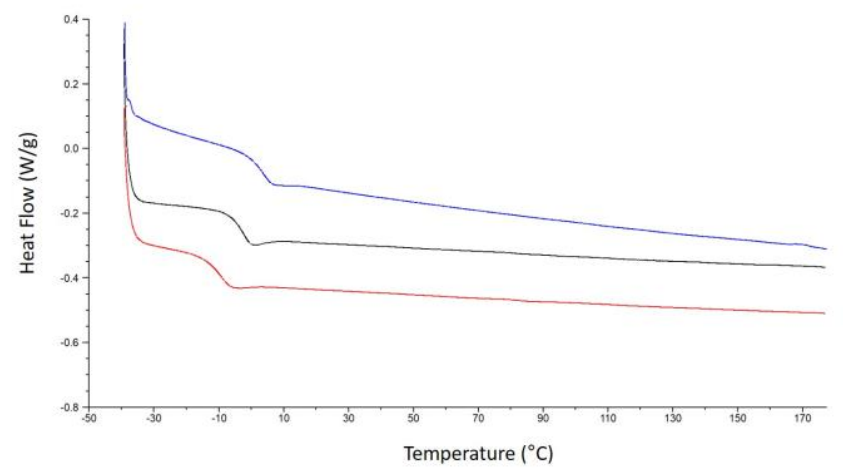

Figure 9. Overlay of DSC second heating cycles of $\mathrm{PHU}_{13}$ (magnetic stirring, red), $\mathrm{PHU}_{14}$ (mechanical stirring, black) and $\mathrm{PHU}_{10}$ (reactive extrusion, blue)

Additionally, the Seb-bCC-ester based PHUs prepared from magnetic and mechanical stirring were found to be soluble in classical organic solvents. ${ }^{1} \mathrm{H}$ NMR spectra, FTIR spectra and SEC chromatograms are presented in Figures SI-15, SI-17 and SI-18. According to those data, the carbonate function conversion was found to be incomplete. Despite the fact that SEC traces did not allow accurate molar mass calculation, the solubility of those compounds in DMF and DMSO could be related to their lower molar mass, in comparison to PHUs synthesized by reactive extrusion.

Table 3. Seb-bCC-ester based PHUs synthesized at $90^{\circ} \mathrm{C}$ by mechanical or magnetic stirring and associated thermal properties.

\begin{tabular}{|c|c|c|c|}
\hline Process & PHU & DA & $\begin{array}{c}\text { Polymeri- } \\
\text { zation } \\
\text { time }\end{array}$ \\
\hline
\end{tabular}




\begin{tabular}{l|lllll}
$\begin{array}{l}\text { Magnetic } \\
\text { Stirring }\end{array}$ & 13 & $6 \mathrm{DA}$ & h & 235 & -10 \\
$\begin{array}{l}\text { Mechanical } \\
\text { Mech }\end{array}$ & 14 & $6 \mathrm{DA}$ & $\mathrm{h}$ & 242 & -3
\end{tabular}

Stirring

This observation is consistent with the above discussed, $T_{g}$ s trend, according to Flory Fox relation between $M_{n}$ and $T_{g}$ for a given polymer.

The above results show that an efficient blending and homogenization of the reaction medium is required during the whole polymerization to reach a full carbonate conversion. Indeed, in the case of bulk polymerization between bisCCs and amines, the highly viscous systems require an efficient shearing along with the polymerization in order to ensure the diffusion of reactive species.

In addition to the excellent mixing capacity brought by the reactive extrusion process on highly viscous systems like Seb-bCCester based PHUs, allowing short reaction time and minimizing side reactions, the second added value to such process is the capacity to produce "ready to use" materials.

\section{Toward reactive extrusion for 'Non-Activated' bisCC: a step further}

Finally, the reactive extrusion process was tested on a non-activated (bis)amide-carbonate. This bisCC was synthesized from methyl undecenoate and 6DA, leading to Und-6DA and then to Und-6DA-bisCC by $\mathrm{CO}_{2}$ fixation, after an epoxidation step (Und6DA-bisEpox), according to the protocol published by Maisonneuve et al. ${ }^{6}$. Detailed ${ }^{1} \mathrm{H}$ NMR spectra of Und-6DA,<smiles>O=C(CCCCCCCC1COC(=O)O1)NCCCCCCNC(=O)CCCCCCC1COC(=O)O1</smiles>

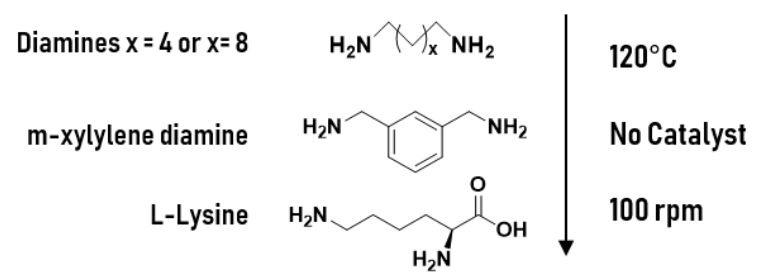

\section{PHUs $_{15-18}$}

Figure 10. Polyaddition reaction of Und-6DA-bisCC with different diamines, leading to $\mathrm{PHU}_{15-18}$

Und-6DA-bisEpox intermediate and final Und-6DA-bisCC, as well as detailed synthetic procedures, can be found in Figures SI21, SI-22, SI-23. In the case of this bisCC, the cyclic carbonate is substituted by a long alkyl chain. As demonstrated by Garipov et al., the aliphatic chain in alpha position of the carbonate ring exhibits an electron releasing effect which decreases the electrophily of the carbonyl and thus reduces the reactivity of the monomer ${ }^{28}$. Besides, the presence of two amide groups which are interesting moieties to bring mechanical properties to the PHUs, turn these reactants in very hard to process materials. Indeed, the carbonated monomer exhibits high melting points $\left(114^{\circ} \mathrm{C}\right)$ and is difficult to solubilize in common solvents. $4^{6}$

Polymerizing by reactive extrusion Und-6DA-bisCC with 6DA, 10DA, m-xylylene diamine or L-Lysine as comonomers, led to $\mathrm{PHU}_{15-18}$, as reported in Table 4 and Figure 10. Due to the melting points of amide-based CCs, polymerizations were performed over $120^{\circ} \mathrm{C}$ in order to ensure a complete melting $\left(220^{\circ} \mathrm{C}\right.$ for $\left.\mathrm{PHU}_{18}\right)$. No catalysts were added and the screw rotation speed was kept at $100 \mathrm{rpm}$.

As the final PHUs so-formed were found very difficult to solubilize in classical solvents, FTIR spectroscopy was used to analyze their structure. The stacked spectra displayed in Figure 11 show that complete conversion of cyclic carbonate functions was obtained for $\mathrm{PHU}_{15}$, synthesized obtained with $10 \mathrm{DA}$ as co-monomer, and very low amount of carbonate functions are remaining in the other cases (small remaining band at $v=1782 \mathrm{~cm}^{-1}$ ). Besides, on these spectra, is also visible the band corresponding to urethane moiety $\left(v=1685 \mathrm{~cm}^{-1}\right)$ and the one appearing at $v=1632 \mathrm{~cm}^{-1}$ attributed to the amide bond. 


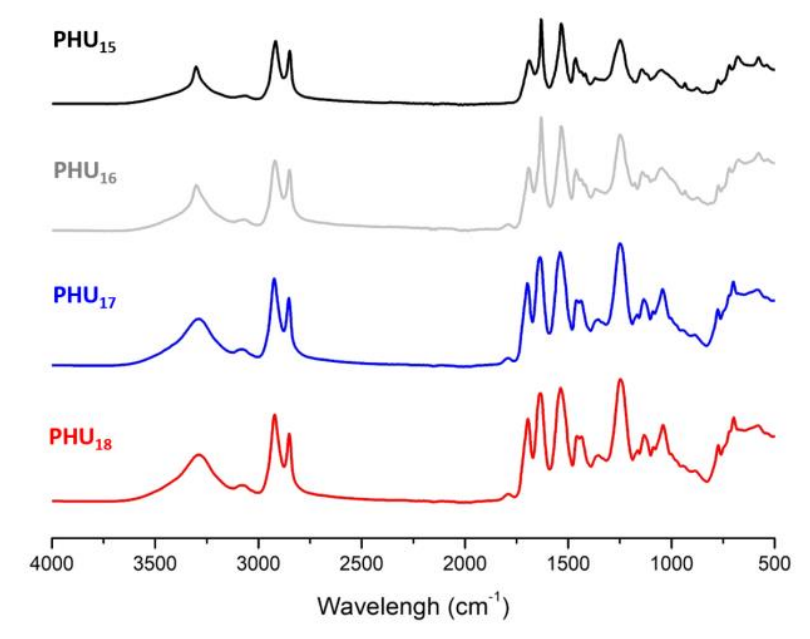

Figure 11. Stacked FTIR spectra of PHU $_{17}$ (Grey), PHU $_{16}$ (blue), PHU $_{15}$ (Red) and PHU $_{18}$ (black).

Figure 12 displays the $\Delta \mathrm{P}=\mathrm{f}(\mathrm{t})$ profiles, obtained for each system during the polymerizations. As clearly visible on the Figure, the pressure values reached in the specific case of Und-6DA-bisCC were extremely high, up to 40 bars for $\mathrm{PHU}_{15,16,17}$ and even to 120 bars in the case of L-Lysine-based PHU $\left(\mathrm{PHU}_{18}\right)$. These very high pressures demonstrate the drastic increase in viscosity, correlated with the fast molar mass build-up and the extremely cohesive feature of this amide type bisCC. Temperature and time-related $\Delta \mathrm{P}$ profiles can be seen in Figure SI-24. In the cases

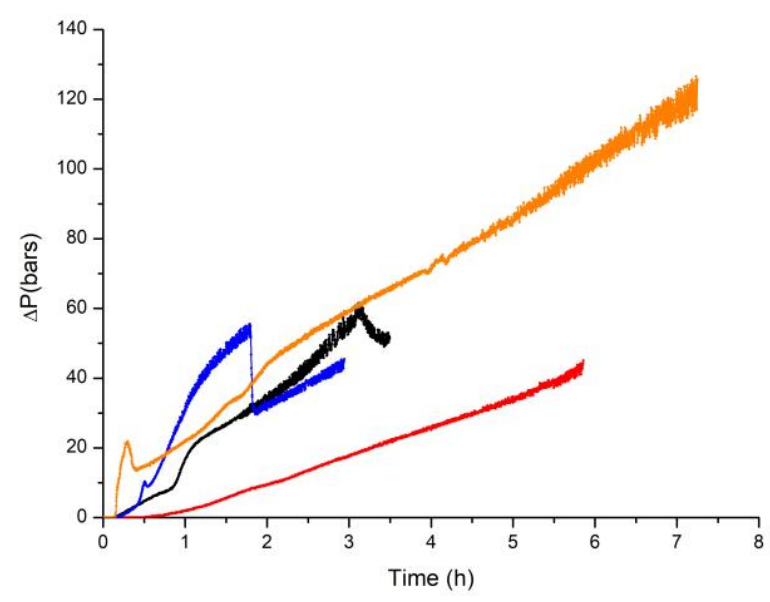

Figure 12. $\Delta \mathrm{P}=\mathrm{f}(\mathrm{t})$ profiles of $\mathrm{PHU}_{17}$ (Red), $\mathrm{PHU}_{16}\left(\right.$ Blue), $\mathrm{PHU}_{15}$ (Black) and $\mathrm{PHU}_{18}($ Orange). (Drops in pressure corresponds to polymerization temperature increase)

\section{Thermal properties of Und-6DA-bisCC based PHUs}

As previously mentioned, the Und-6DA-bisCC based PHUs were found difficult to solubilize in classical solvents, preventing size exclusion chromatography analysis. Furthermore, a recent study published by Zhang et al. explained that segmented PHUs containing amide linkages may interact with common SEC columns, thus making molar masses characterization by SEC not feasible. ${ }^{47}$ Nevertheless, thermal analysis (DSC and TGA) have been performed on these PHUs and results are reported in Table 4. Delebecq et al. demonstrated that electronic effects of $\alpha-\beta$ substituents on cylic carbonates not only increase their reactivity but also impact the thermal degradation of the final PU, in a negative way..$^{8}$ This relevant conclusion explains the higher thermal stabilities, up to $325^{\circ} \mathrm{C}$, measured for the non-activated Und-6DA-bisCC based PHUs, compared to previous PHUs synthesized from ether and ester activated carbonates. TGA and DSC curves of $\mathrm{PHU}_{15-18}$ can be seen in Figure SI-25 and SI-26.

Table 4. Und-6DA-based PHUs and associated thermal properties.

\begin{tabular}{c|ccccc} 
PHU & Diamines & $\begin{array}{c}\text { Time } \\
(\mathbf{h})\end{array}$ & $\begin{array}{c}\mathrm{T}_{\mathrm{g}} \mathbf{a} \\
\left({ }^{\circ} \mathbf{C}\right)\end{array}$ & $\mathbf{T}_{\mathbf{m}}{ }^{\mathrm{a}}\left({ }^{\circ} \mathbf{C}\right)$ & $\begin{array}{c}\mathbf{T}_{\mathbf{d} 5 \%}{ }^{\mathbf{c}} \\
\left({ }^{\circ} \mathbf{C}\right)\end{array}$ \\
\hline 15 & $10 D A$ & $3 \mathrm{~h} 30$ & 17 & $100 / 140^{\mathrm{b}}$ & 263
\end{tabular}




\begin{tabular}{c|ccccc}
16 & $6 \mathrm{DA}$ & $3 \mathrm{~h}$ & 22 & $95^{\mathrm{b}}$ & 266 \\
17 & m-xylylene & $6 \mathrm{~h}$ & 32 & -- & 265 \\
18 & L-Lysine & $7 \mathrm{~h} 15$ & 36 & -- & 325
\end{tabular}

${ }^{\text {a }}$ Determined by DSC second heating cycle at $10^{\circ} \mathrm{C} / \mathrm{min}$. ${ }^{\mathrm{b}}$ With crystallization upon heating. ${ }^{\mathrm{c}}$ Determined by $\mathrm{TGA}$ at $10^{\circ} \mathrm{C} / \mathrm{min}$ under $\mathrm{N}_{2}$.

As expected, amide linkages bring rigidity to the materials and $\mathrm{T}_{\mathrm{g} S}$ values for Und-6DA-bisCC based PHUs, were found to be higher than those of DGDC and Seb-bCC-ester based PHUs (up to $36^{\circ} \mathrm{C}$ ).

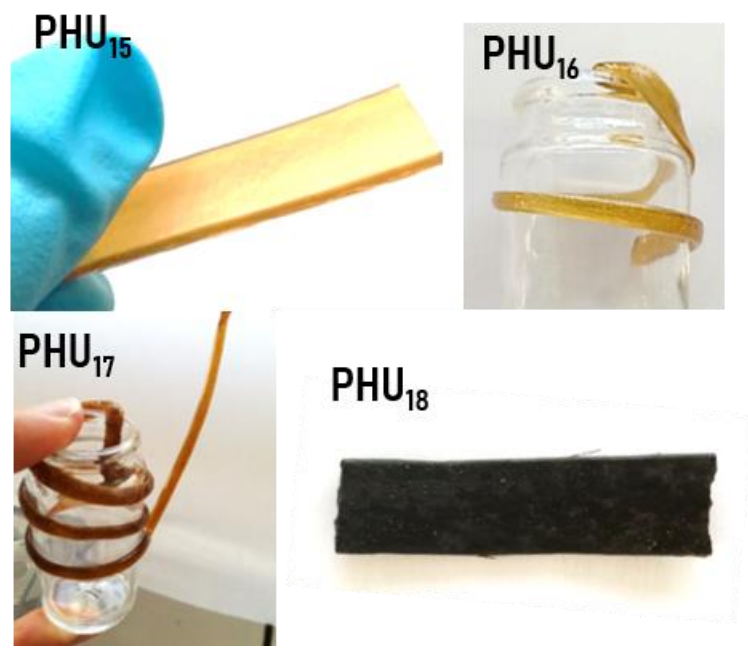

Figure 13. Pictures of $\mathrm{PHU}_{15-18}$, directly obtained from the reactor of the extruder.

Furthermore, the highest thermal stability $\left(\mathrm{T}_{\mathrm{d} 5 \%}=325^{\circ} \mathrm{C}\right)$ was achieved with safe, biobased, but hard to process L-Lysine $(\mathrm{mp}=$ $215^{\circ} \mathrm{C}$ ). Once again, this result highlights the benefit brought by reactive extrusion on melt polymerizations of high melting points and/or very cohesive derivatives.

Finally, as shown in Figure 13, Und-6DA-based PHUs were extruded and obtained as homogeneous materials and this in much shorter reaction times compared to results previously published by Maisonneuve et al. ( 3 to 7 hours vs few days). ${ }^{6}$ In their study, the authors deals with the synthesis and polymerization of a series of fatty acid-based bis-cyclic carbonates bearing amide linkages. Bulk polymerizations were performed at $120^{\circ} \mathrm{C}$ or $140^{\circ} \mathrm{C}$, allowing the relevant comparison with $\mathrm{PHU}_{15} \mathrm{PHU}_{16}$ and $\mathrm{PHU}_{17}$. In order to reach high carbonate functions conversion, reactions times within between $5 \mathrm{~h}$ and 7 days were needed. The low reaction rate is due to the very slow mobility of reactants, trapped in the very viscous and cohesive hydroxyurethane-amide systems. The above results thus demonstrate that even non-activated carbonates can be polymerized in short reaction times with a palette of diamines exhibiting various structures.

\section{Conclusion}

Different PHUs have been synthesized from bio-based activated and non-activated biscyclic carbonates, Seb-bCC-ester, DGDC and Und-6DA-bisCC, through reactive extrusion process.

Complete conversions of reactive carbonate functions were obtained in the case of DGDC and Seb-bCC-ester monomers, in short reaction times (hours instead of days), without the need of excessive heating. Those mild conditions and the shorter reaction times prevent the formation of by-products, as no urea was created with DGDC and very low amounts of urea and amide were detected in the case of Seb-bCC-ester based PHUs. Regarding these last polymers, the consistency of $\mathrm{T}_{\mathrm{g}} \mathrm{s}$ and thermal stabilities obtained with different diamines prove the versatility of the process with respect to different monomer structures.

Besides, the processability brought by this system is making possible the synthesis of very dense PHUs materials, even from highly viscous precursors. Finally, this study demonstrates the possibility to polymerize non-activated and amide based bisCCs (such as Und-6DA-bisCC), in reasonable conditions of time and directly leading to rigid materials. Reactive extrusion process stands for a promising tool to push PHU synthesis a bit closer to a viable industrial process.

Finally, one can notice that the mini-extruder equipped with conic twin screws and circulation channel used in this work is not fairly representative of the machines used in industries. Industrial systems are composed of single or twin several meters long coaxial screws. 49 This means that generally residence times are relatively shorts, whereas in this study the polymerizations times last until 7 hours. Nevertheless, the results discussed in this publication are intented to prove the benefit that can be brought by this kind of solvent-free technique on PHU synthesis. Even if polymerization times modulate the final properties and as a consequence 
can be adjusted according to targetted applications, further optimization is needed to fit with industrials equipments. As a perspective, the polymerization by reactive extrusion of thermosets PHUs will be investigated, in order to evaluate possible shorter polymerization times.

\section{ASSOCIATED CONTENT}

Additional ${ }^{1} \mathrm{H}$ NMR spectra, FTIR spectra of PHUs and/or bis-cyclic carboantes. Thermal analysis (DSC and TGA) traces of PHUs. SEC comparison of DGDC-10DA-based PHUs syntheseized through magnetic stirring, mechanical stirring and reactive extrusion. Additionnal analysis ( $\left.{ }^{1} \mathrm{HNMR}, \mathrm{FTIR}, \mathrm{SEC}\right)$ on Seb-bCC-ester-based PHUs synthesizez through magnetic and mechanical stirring. $(\Delta \mathrm{P}, \mathrm{T})=\mathrm{f}(\mathrm{t})$ profiles for Und-6DA-based PHUs.

\section{AUTHOR INFORMATION}

\section{Corresponding author}

*E-mail: cramail@enscbp.fr

\section{ACKNOWLEDGEMENTS}

The authors gratefully acknowledge the financial support from Region Nouvelle Aquitaine and ITERG. The authors sincerely thank ITERG (French Institute for Fats and Oils) for providing chemical samples.

\section{REFERENCES}

Bayer, O. Das Di-lsocyanat-Poluadditionsverfahren (Polyurethane). Angew. Chemie 59, 257-272 (1947).

Nohra, B., Candy, L., Guerin, C. \& Raoul, Y. From Petrochemical Polyurethanes to Biobased Polyhydroxyurethanes. (2013). doi:10.1021/ma400197c

Rokicki, G., Parzuchowski, P. G. \& Mazurek, M. Non-isocyanate polyurethanes: Synthesis, properties, and applications. Polymers for Advanced Technologies 26, 707-761 (2015).

Marion, P. et al. Sustainable chemistry: How to produce better and more from less? Green Chem. 19, 4973-4989 (2017). Kayode, F. A Review of Vegetable Oil-Based Polymers: Synthesis and Applications. Open J. Polym. Chem. 5, 34-40 (2015).

Rose, M. \& Palkovits, R. Isosorbide as a renewable platform chemical for versatile applications-quo vadis? ChemSusChem 5, 167-176 (2012). Galbis, J. A., García-Martín, M. D. G., De Paz, M. V. \& Galbis, E. Synthetic Polymers from Sugar-Based Monomers. Chem. Rev. 116, 16oo$1636(2016)$

Wilbon, P. A., Chu, F. \& Tang, C. Progress in Renewable Polymers from Natural. Macromol. Rapid Commun. 34, 32-43 (2013).

Wang, H., Pu, Y., Ragauskas, A. \& Yang, B. From lignin to valuable products-strategies, challenges, and prospects. Bioresour. Technol. 271, 449-461 (2019).

Mokhtari, C., Malek, F., Manseri, A., Caillol, S. \& Negrell, C. Reactive jojoba and castor oils-based cyclic carbonates for biobased polyhydroxyurethanes. Eur. Polym. J. 113, 18-28 (2019).

Carré, C., Bonnet, L. \& Avérous, L. Original biobased nonisocyanate polyurethanes: Solvent- and catalyst-free synthesis, thermal properties and rheological behaviour. RSC Adv. 4, 54018-54025 (2014).

Maisonneuve, L., Lamarzelle, O., Rix, E., Grau, E. \& Cramail, H. Isocyanate-Free Routes to Polyurethanes and Poly ( hydroxy Urethane ) s. (2015). doi:10.1021/acs.chemrev.5boo355

Lamarzelle, O. et al. Activated lipidic cyclic carbonates for non-isocyanate polyurethane synthesis. Polym. Chem. 7, 1439-1451 (2016). Steblyanko, A., Choi, W., Sanda,
Synthesis. 38, 2375-2380 (2000).

Maisonneuve, L. et al. Novel green fatty acid-based bis-cyclic carbonates for the synthesis of isocyanate-free poly(hydroxyurethane amide)s. RSC Adv. 4, 25795-25803 (2014).

Bigot, S. et al. Undecylenic acid : A tunable bio-based synthon for materials applications. Eur. Polym. J. 74, 26-37 (2016).

Camara, F. et al. Reactivity of secondary amines for the synthesis of non-isocyanate polyurethanes. Eur. Polym. J. 55, 17-26 (2014).

Janvier M., Paul-Henri Ducrot, and F. A. Isocyanate-Free Synthesis and Characterization of Renewable Poly(hydroxy)urethanes from Syringaresinol. (2017). doi:10.1021/acssuschemeng.7bo1271

Maisonneuve, L., Wirotius, A. L., Alfos, C., Grau, E. \& Cramail, H. Fatty acid-based (bis) 6-membered cyclic carbonates as efficient isocyanate free poly(hydroxyurethane) precursors. Polym. Chem. 5, 6142-6147 (2014).

Sayed, M. Multi-steps Green Process for Synthesis of Six-Membered Functional Cyclic Carbonate from Trimethylolpropane by Lipase Catalyzed Methacrylation and Carbonation , and Thermal Cyclization. 12-14 (2015). doi:10.1002/btpr.2201

Fortman, D. J., Brutman, J. P., Cramer, C. J., Hillmyer, M. A. \& Dichtel, W. R. Mechanically Activated, Catalyst-Free Polyhydroxyurethane Vitrimers. J. Am. Chem. Soc. 137, 14019-14022 (2015).

Tryznowski, M., Zołek-Tryznowska, Z., widerska, A. \& Parzuchowski, P. G. Synthesis, characterization and reactivity of a six-membered cyclic glycerol carbonate bearing a free hydroxyl group. Green Chem. 18, 802-807 (2016).

Sanders, D. P. et al. Synthesis of functionalized cyclic carbonate monomers using a versatile pentafluorophenyl carbonate intermediate. Polym. Chem. 5, 327-329 (2014).

Tomita, H., Sanda, F. \& Endo, T. Polyaddition of bis(seven-membered cyclic carbonate) with diamines: A novel and efficient synthetic method for polyhydroxyurethanes. J. Polym. Sci. Part A Polym. Chem. 39, 4091-4100 (2001).

Tomita, H., Sanda, F. \& Endo, T. Polyaddition Behavior of bis ( Five- and Six-Membered Cyclic Carbonate ) s with Diamine. 86o-867 (2000).

Yuen, A. et al. Room temperature synthesis of non-isocyanate polyurethanes (NIPUs) using highly reactive N-substituted 8-membered cyclic carbonates. Polym. Chem. 7, 2105-2111 (2016). 
Tomita, H., Sanda, F. \& Endo, T. Model reaction for the synthesis of polyhydroxyurethanes from cyclic carbonates with amines: Substituent effect on the reactivity and selectivity of ring-opening direction in the reaction of five-membered cyclic carbonates with amine. J. Polym. Sci. Part A Polym. Chem. 39, 3678-3685 (2001).

28. Garipov, R. M. et al. Reactivity of Cyclocarbonate Groups in Modified Epoxy-Amine Compositions. Dokl. Phys. Chem. 393, 289-292 (2003). Lamarzelle, O. et al. Activated lipidic cyclic carbonates for non-isocyanate polyurethane synthesis. Polym. Chem. 7, 1439-1451 (2016).

He, Y., Goel, V., Keul, H. \& Möller, M. Synthesis, characterization, and selectivity of bifunctional couplers. Macromol. Chem. Phys. 211, 2366-2381 (2010).

Cornille, A. et al. A study of cyclic carbonate aminolysis at room temperature: Effect of cyclic carbonate structures and solvents on polyhydroxyurethane synthesis. Polym. Chem. 8, 592-604 (2017).

Cornille, A., Auvergne, R., Figovsky, O., Boutevin, B. \& Caillol, S. A perspective approach to sustainable routes for non-isocyanate polyurethanes. 87, 535-552 (2017).

Besse, V. et al. How to explain low molar masses in PolyHydroxyUrethanes (PHUs). Eur. Polym. J. 71, 1-11 (2015). Thermoplastics and Thermoplastic Elastomers. Macromolecules 50, 2296-2303 (2017). Schimpf, V., Max, J. B., Stolz, B., Heck, B. \& Mülhaupt, R. Semicrystalline Non-Isocyanate Polyhydroxyurethanes as Thermoplastics and Thermoplastic Elastomers and Their Use in 3D Printing by Fused Filament Fabrication. Macromolecules 52, 320-331 (2019). Tryznowski, M., widerska, A., Zołek-Tryznowska, Z., Gołofit, T. \& Parzuchowski, P. G.
environmentally friendly non-isocyanate polyurethanes. Polymer (Guildf). 8o, 228-236 (2015). Weckhuysen, B. M. \& Bruijnincx, P. C. A. carbonate synthesis †. 1605-1618 (2016). doi:10.1039/c5gco2046h

38. Van Velthoven, J. L. J., Gootjes, L., Van Es, D. S., Noordover, B. A. J. \& Meuldijk, J. Poly(hydroxy urethane)s based on renewable diglycerol dicarbonate. Eur. Polym. J. 70, 125-135 (2015).

Stewart, J. A., Weckhuysen, B. M. \& Bruijnincx, P. C. A. Reusable Mg-Al hydrotalcites for the catalytic synthesis of diglycerol dicarbonate from diglycerol and dimethyl carbonate. Catal. Today 257, 274-280 (2015). Bossion, A. et al. Unexpected Synthesis of Segmented Poly(hydroxyurea-urethane)s from Dicyclic Carbonates and Diamines by Organocatalysis. Macromolecules 51, 5556-5566 (2018).

Benyahya, S., Boutevin, B., Caillol, S., Lapinte, V. \& Habas, J. P. Optimization of the synthesis of polyhydroxyurethanes using dynamic rheometry. Polym. Int. 61, 918-925 (2012).

Blain, M., Auvergne, R., Benazet, D., Caillol, S. \& Andrioletti, B. Rational investigations in the ring opening of cyclic carbonates by amines †. 4286-4291 (2014). doi:10.1039/c4gco1032a

Blain, M. et al. Hydrogen bonds prevent obtaining high molar mass PHUs. J. Appl. Polym. Sci. 134, 44958 (2017).

Podzimek, S. Molar mass distribution by size exclusion chromatography: Comparison of multi-angle light scattering and universal calibration. 47561, 1-10 (2019).

Benyahya, S., Habas, J. P., Auvergne, R., Lapinte, V. \& Caillol, S. Structure-property relationships in polyhydroxyurethanes produced from terephthaloyl dicyclocarbonate with various polyamines. Polym. Int. 61, 1666-1674 (2012).

Maisonneuve, L. et al. Novel green fatty acid-based bis-cyclic carbonates for the synthesis of isocyanate-free poly(hydroxyurethane amide)s. RSC Adv. 4, 25795-25803 (2014).

Zhang, K. et al. Non-isocyanate poly(amide-hydroxyurethane)s from sustainable resources. Green Chem. 18, 4667-4681 (2016). 113, 8o-118 (2013).

Günter Beyer \& Hopmann, C. Reactive Extrusion. Reactive Polymers: Fundamentals and Applications (2018). doi:10.1016/B978-0-12-8145098.00015-4 


\section{Benefit of the reactive extrusion in the course of poly(hydroxy)urethanes synthesis by aminolysis of cyclic carbonates}

Fiona Magliozzi ${ }^{1}$, Guillaume Chollet ${ }^{2}$, Etienne Grau ${ }^{1}$ and Henri Cramail1,*

${ }^{1}$ Univ. Bordeaux, CNRS, Bordeaux INP, LCPO, UMR 5629, F-336oo, Pessac, France ${ }^{2}$ ITERG, 11 Rue Gaspard Monge, 33610 Canéjan France

Pages : 20

Figures : 26

Tables : 1 


\section{TABLE OF CONTENTS}

\section{DGDC-based PHUs}

Figure SI-1 $\quad{ }^{1}$ HNMR spectrum of DGDC, in DMSO-d6, RT, 64 scans

Figure SI-2 $\quad{ }^{1} \mathrm{HNMR}$ spectrum of $\mathrm{PHU}_{1}$, DGDC/6DA/Reactive Extrusion, 1024 scans

Figure SI-3 $\quad{ }^{1} \mathrm{HNMR}$ spectrum of $\mathrm{PHU}_{2}$, DGDC/10DA/Reactive Extrusion, 1024 scans

Figure SI-4 FTIR spectra of $\mathrm{PHU}_{1}$ and $\mathrm{PHU}_{2}$

Figure SI-5 Stacked ${ }^{1}$ HNMR spectra of DGDC-based PHUs synthesized from 6DA and 10DA through magnetic stirring.

Figure SI-6 Stacked ${ }^{1}$ HNMR spectra of DGDC-based PHUs synthesized from 6DA and 10DA through mechanical stirring.

Figure SI-7 SEC traces in DMF, DGDC and 10DA based PHUs synthesized through different processes: $\mathrm{PHU}_{2}$ (reactive extrusion), $\mathrm{PHU}_{4}$ (magnetic stirring) and $\mathrm{PHU}_{6}$ (mechanical stirring)

Figure SI-8 Overlay of TGA traces of DGDC/10DA-based PHUs, synthesized through magnetic stirring $\left(\mathrm{PHU}_{4}\right)$, mechanical stirring $\left(\mathrm{PHU}_{6}\right)$ and reactive extrusion $\left(\mathrm{PHU}_{2}\right)$

Figure SI-9 Overlay of DSC second heating cycle of DGDC/6DA-based PHUs. PHU (reactive extrusion), $\mathrm{PHU}_{3}$ (magnetic stirring), $\mathrm{PHU}_{5}$ (mechanical stirring)

Figure SI-10 Overlay of DSC second heating cycle of DGDC/10DA-based PHUs. $\mathrm{PHU}_{2}$ (reactive extrusion), $\mathrm{PHU}_{4}$ (magnetic stirring), $\mathrm{PHU}_{6}$ (mechanical stirring)

\section{Seb-bCC-ester-based PHUs}

Figure SI-11 $\quad{ }^{1}$ HNMR spectrum of Seb-bCC-ester 
Figure SI-12 Zoom between 5.6 and $8.1 \mathrm{ppm}$ on ${ }^{1} \mathrm{HNMR}$ spectrum, of the soluble fraction of $\mathrm{PHU}_{10}$ in DMSO-d6 at RT and zoom between 5.6 and 8.1ppm on HR-MAS spectrum, of the insoluble and swollen fraction of PHU 10 in DMSO-d6 at RT

Figure SI-13 Overlay of TGA traces of Seb-bCC-ester-based PHUs synthesized through reactive extrusion. $\mathrm{PHU}_{9}$ (4DA, Red), $\mathrm{PHU}_{10}$ (6DA), $\mathrm{PHU}_{11}$ (10DA) and $\mathrm{PHU}_{12}$ (12DA)

Figure SI-14 Overlay of TGA traces of Seb-bCC-ester-based PHUs synthesized through reactive extrusion $\mathrm{PHU}_{9}$ (4DA), $\mathrm{PHU}_{10}$ (6DA), $\mathrm{PHU}_{11}$ (10DA) and $\mathrm{PHU}_{12}$ (12DA)

Table SI-1 Seb-bCC-ester-based PHUs synthesized from 6DA and 10DA, through mechanical and magnetic stirring, and associated thermal properties

Figure SI-15 $\quad{ }^{1} \mathrm{H}$ NMR spectrum of $\mathrm{PHU}_{14}$, Seb-bCC-ester/6DA/Mechanical stirring and magnetic stirring, in DMSO-d6, RT, 64 scans. *Residual cyclic carbonate. Ratio Amide/Urethane/Urea - Conversion: 1.7/98.3/0 - 89.1\% $\left(\mathrm{PHU}_{14}\right)$, 5.9/94.1/0 - 76.5\% $\left(\mathrm{PHU}_{13}\right)$.

Figure SI-16 $\quad{ }^{1} \mathrm{H}$ NMR spectrum of $\mathrm{PHU}_{19}$, Seb-bCC-ester/10DA/Mechanical stirring and magnetic stirring (bottom), in DMSO-d6, RT, 64 scans. *Residual cyclic carbonate. Ratio Amide/Urethane/Urea - Conversion: 2.2/97.8/0 $85.9 \%\left(\mathrm{PHU}_{19}\right), 1.7 / 98.3 / 0-90.0 \%$

Figure SI-17 FTIR spectra of Seb-bCC-ester-based PHUs. PHU 13 (6DA, magnetic stirring), $\mathrm{PHU}_{14}$ (6DA, mechanical stirring), $\mathrm{PHU}_{19}$ (10DA, magnetic stirring) and $\mathrm{PHU}_{20}(10 \mathrm{DA}$, mechanical stirring).

Figure SI-18 SEC traces in DMF of Seb-bCC-ester-based PHUs synthesized through mechanical stirring from 6DA and 10DA

Figure SI-19 Overlay of DSC second heating cycles of PHU 13 (Seb-bCC-ester/6DA, magnetic stirring) and $\mathrm{PHU}_{14}$ (Seb-bCC-ester/6DA, mechanical stirring)

Figure SI-20 Overlay of DSC second heating cycle of PHU19 (Seb-bCC-ester/10DA, magnetic stirring) and PHU20 (Seb-bCC-ester/10DA, mechanical stirring)

\section{Und-6DA-based PHUs}

Figure SI-21 Epoxidation and carbonation steps leading to Und-DA-bisCC from Und6DA

Figure SI-22 Stacked ${ }^{1}$ HNMR Spectra of Und-6DA and Und-6DA-bisEpoxyde 
Figure SI-23 $\quad{ }^{1}$ HNMR spectrum of Und-6DA-bisCC

Figure SI-24 $\Delta \mathrm{P}=\mathrm{f}(\mathrm{t})$ profiles obtained during synthesis of Und-6DA-based PHUs.

Figure SI-25 Overlay of TGA traces of Und-6DA-bisCC-based PHUs

Figure SI-26 Overlay of DSC second heating cycles of Und-6DA-bisCC-based PHUs 


\section{DGDC Based PHUs}

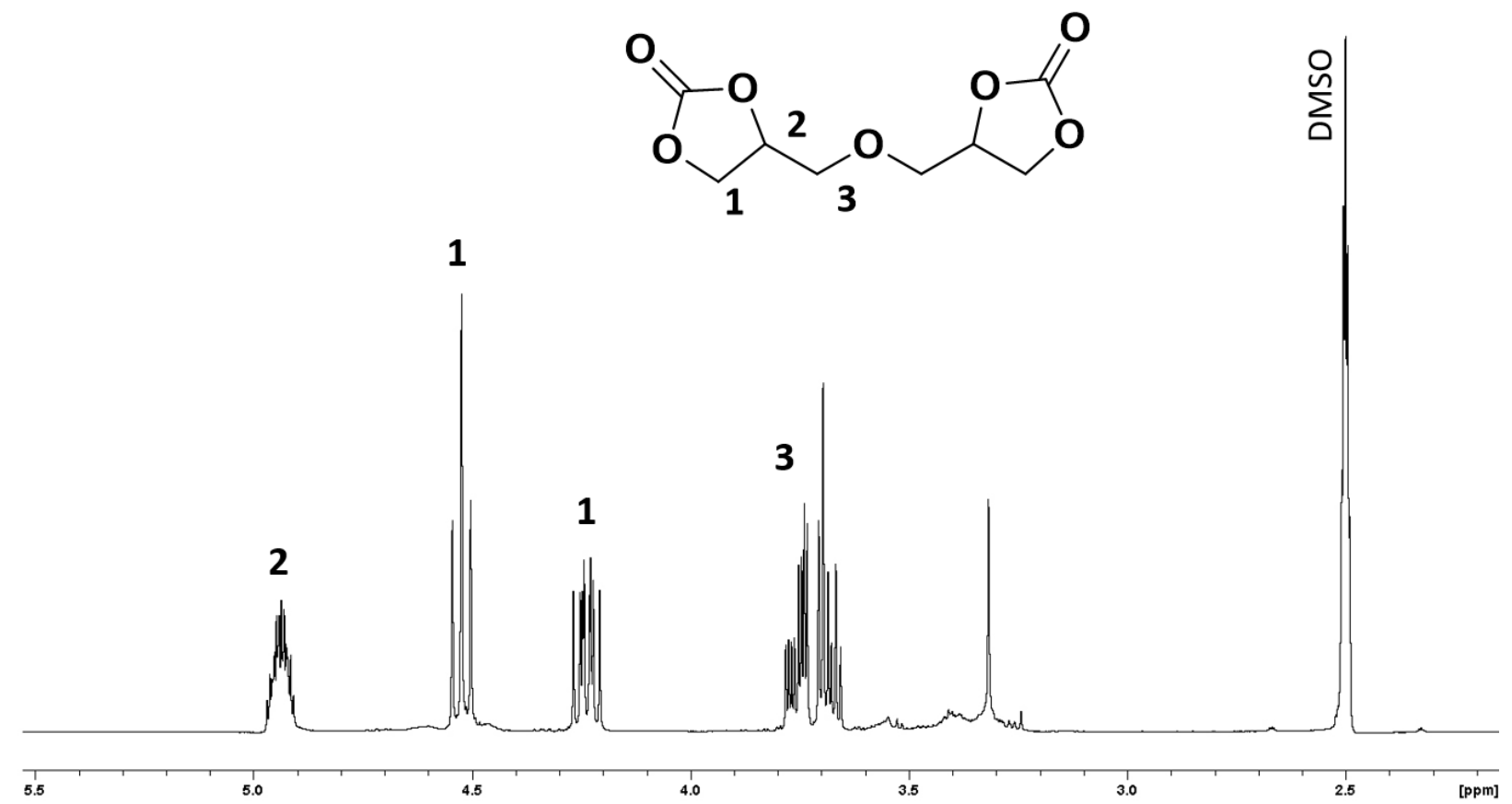

Figure SI-1. 'H NMR spectrum of DGDC, in DMSO-d6, RT, 64 scans 


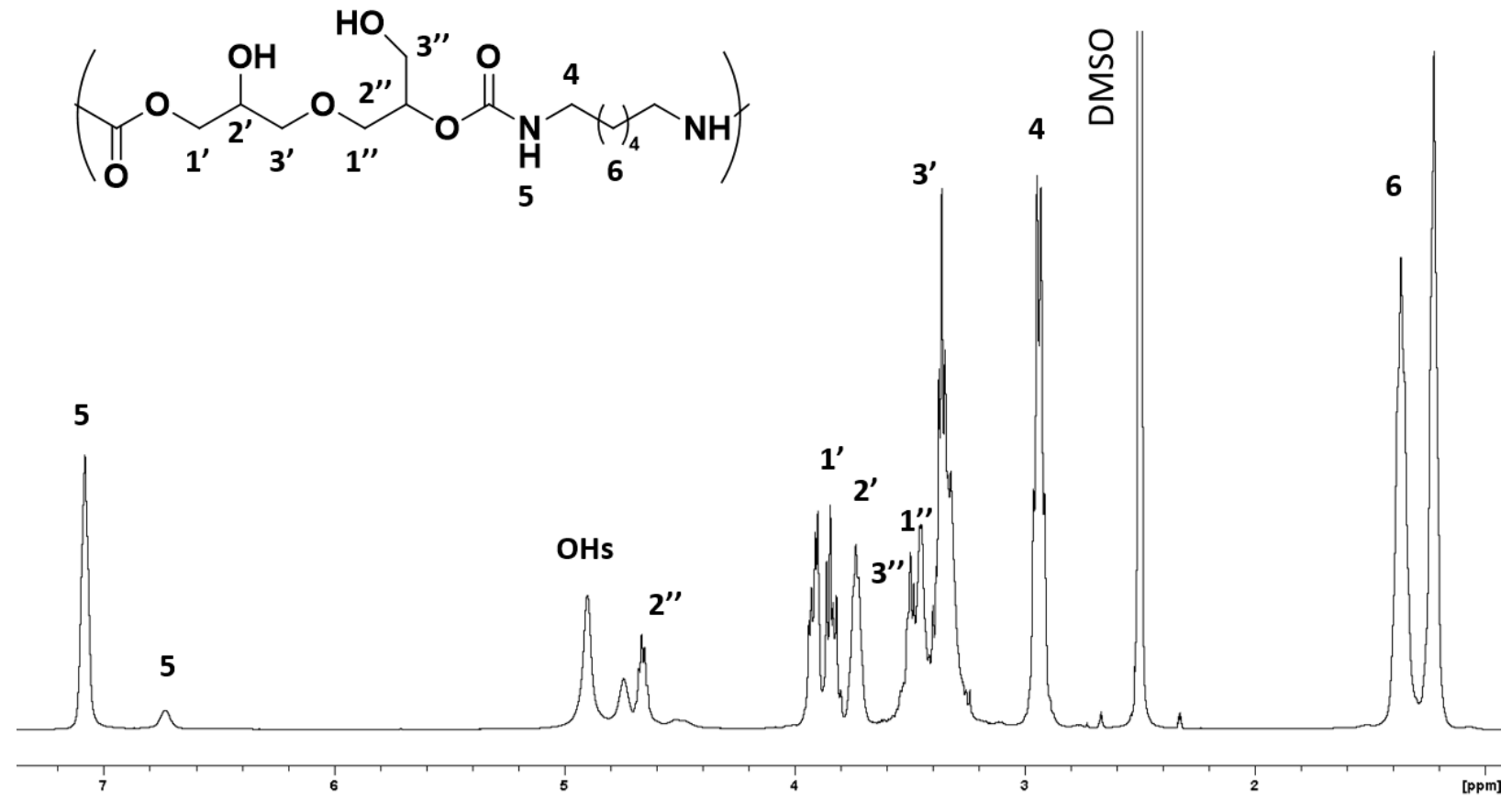

Figure SI-2. ' ${ }^{H}$ NMR spectrum of $\mathrm{PHU}_{1}$, DGDC/6DA/Reactive Extrusion, inDMSO-d6, RT, 1024 scans

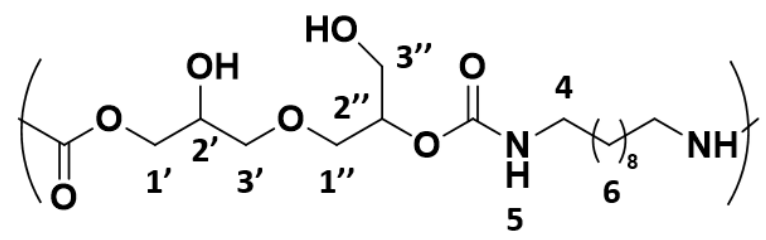

5

5

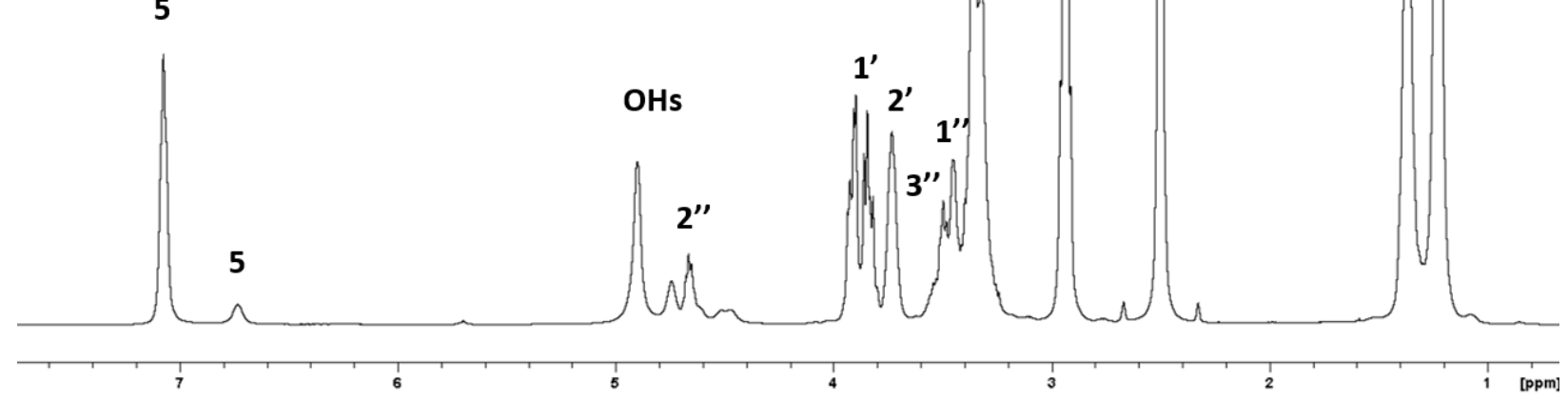

Figure SI-3. ${ }^{\mathrm{H}} \mathrm{NMR}$ spectrum of $\mathrm{PHU}_{2}$, DGDC/1oDA/Reactive Extrusion, inDMSO-d6, RT, 1024 scans 


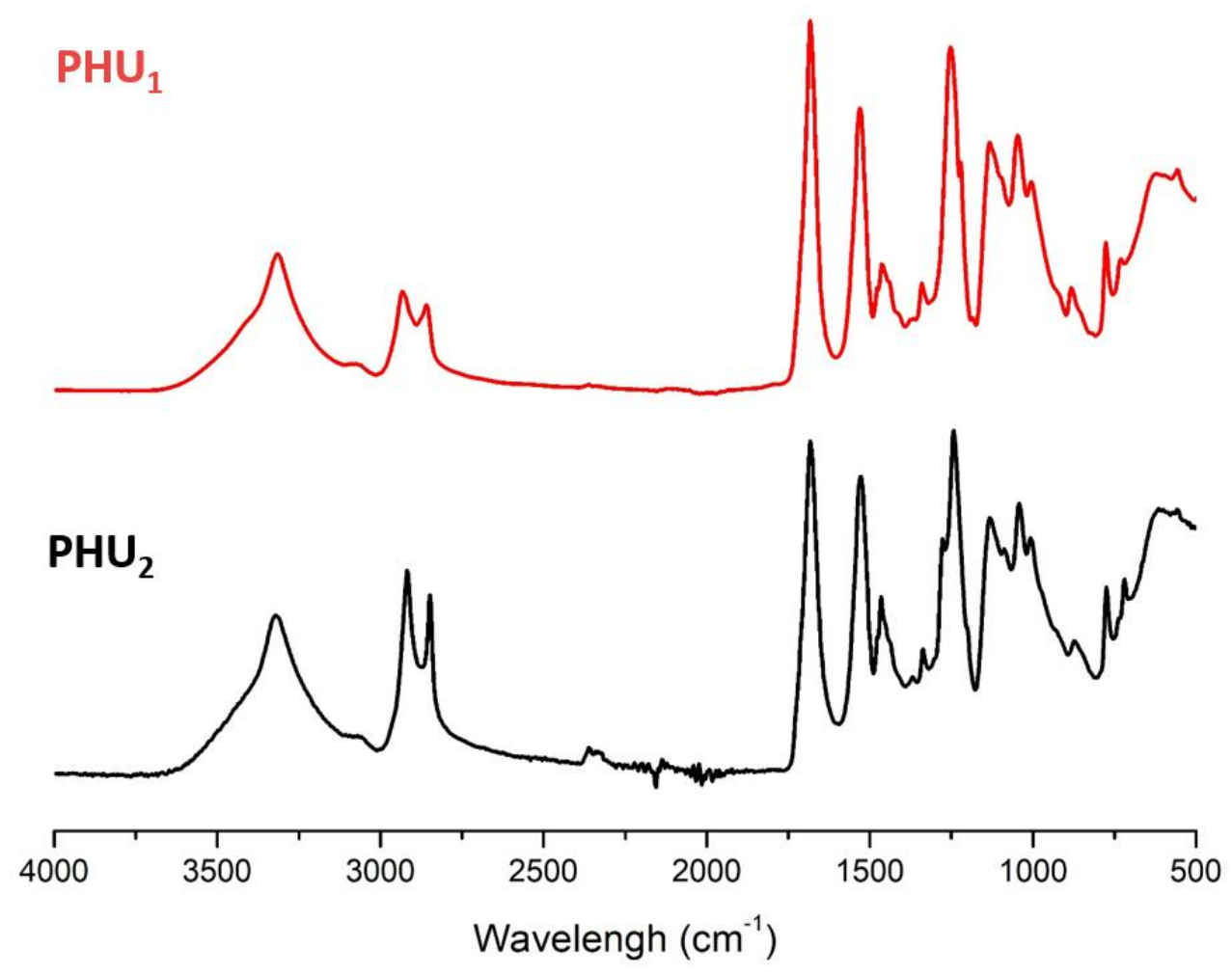

Figure SI-4: FTIR spectra of $\mathrm{PHU}_{1}$ (red) and $\mathrm{PHU}_{2}$ (black)
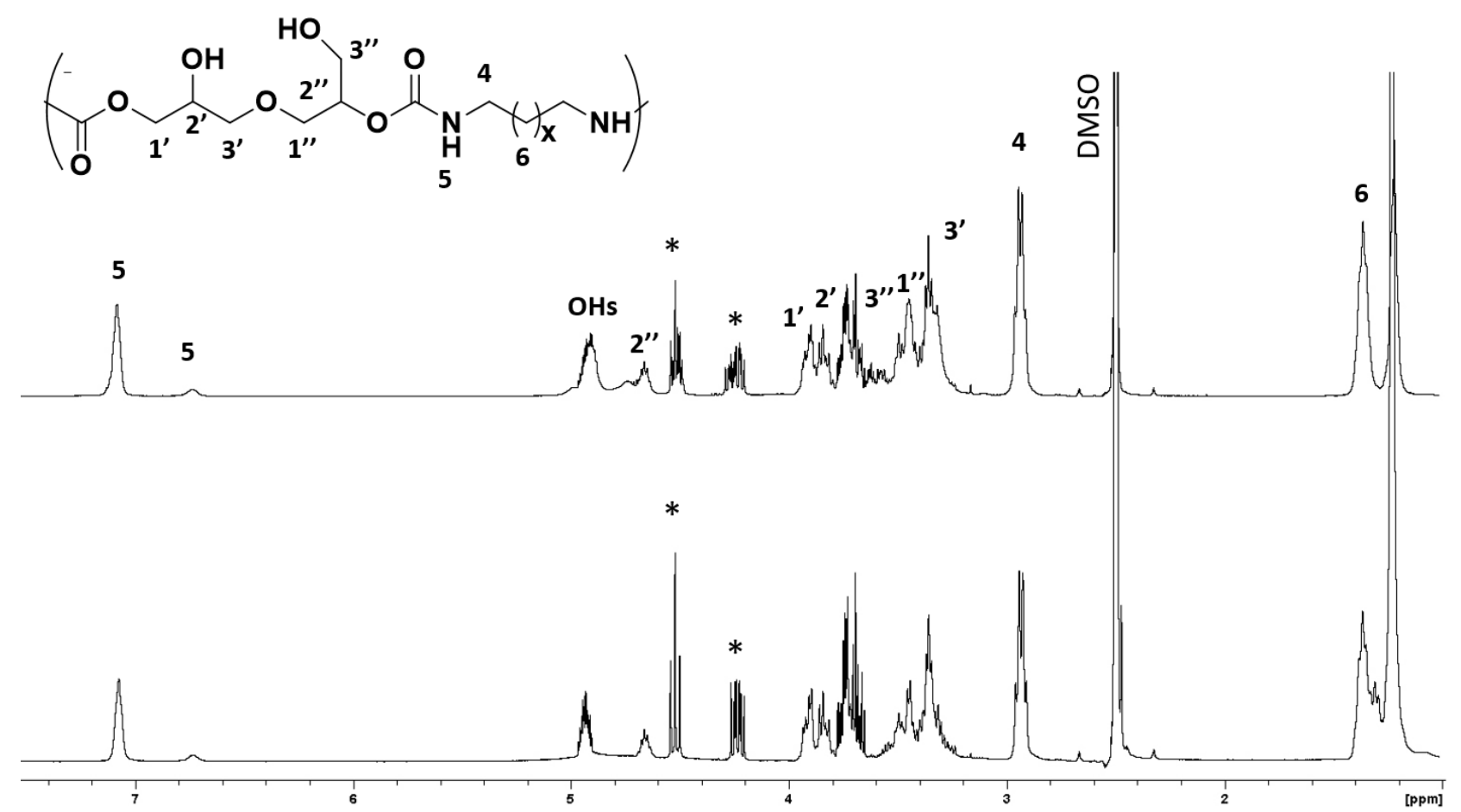

Figure SI-5. Stacked 'H NMR spectra of DGDC-based PHUs synthesized from 6DA and 1oDA through magnetic stirring. $\mathrm{PHU}_{3}$ (top), $\mathrm{PHU}_{4}$ (bottom). * Residual cyclic carbonates 

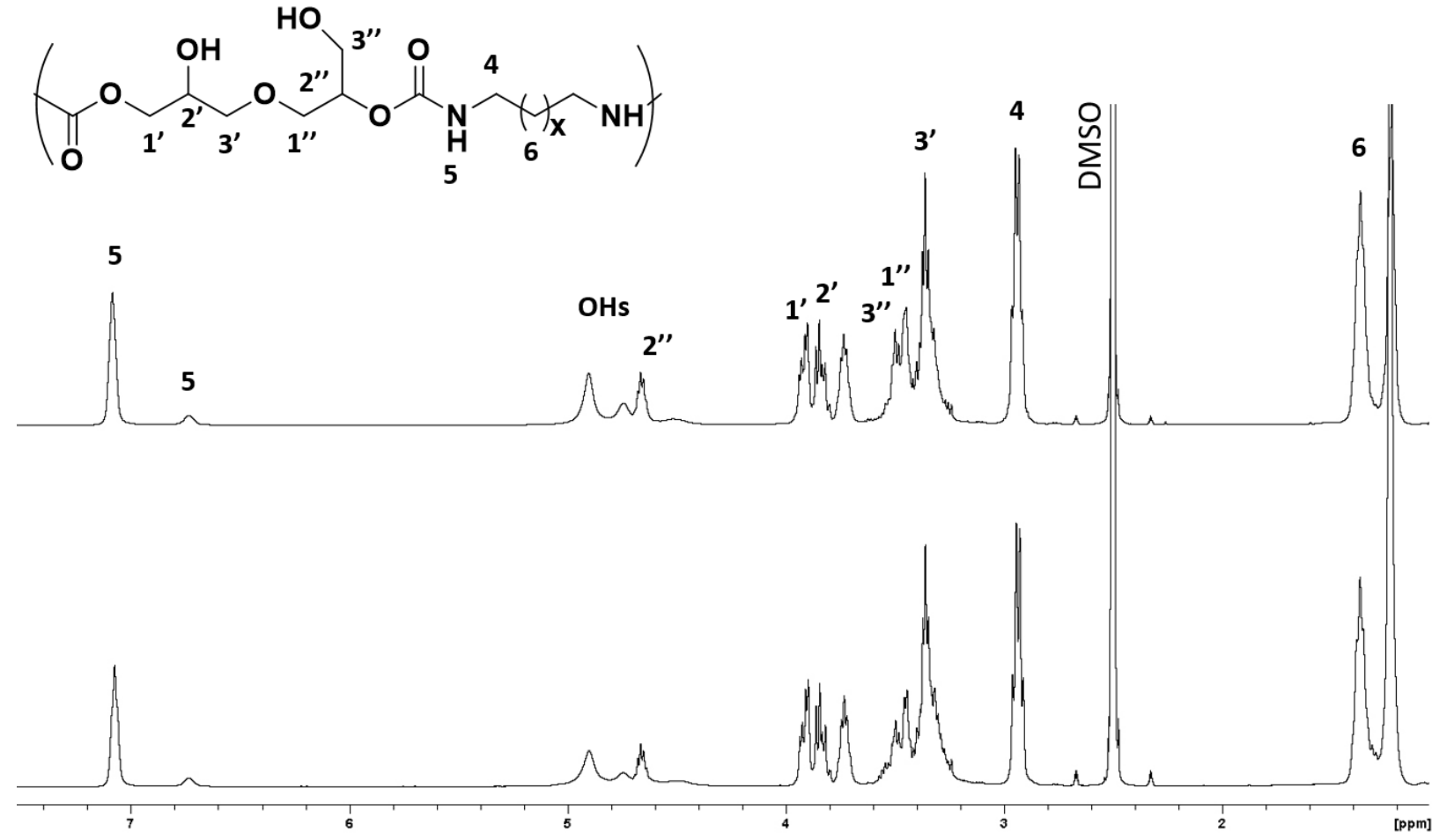

Figure SI-6 Stacked 'H NMR spectra of DGDC-based PHUs synthesized from 6DA and 1oDA through mechanical stirring. $\mathrm{PHU}_{5}$ (top), $\mathrm{PHU}_{6}$ (bottom). * Residual cyclic carbonates
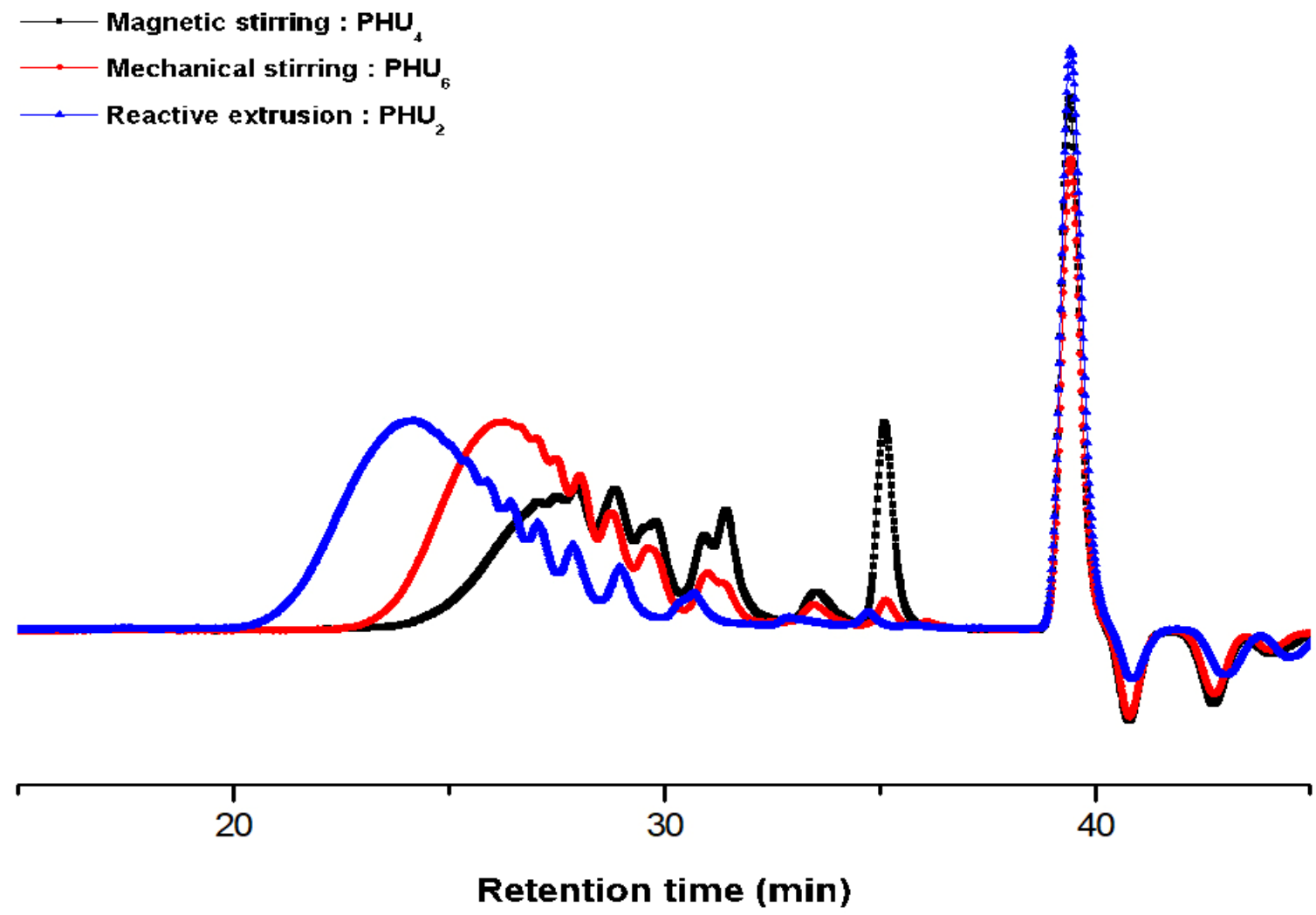

Figure SI-7. SEC traces in DMF, DGDC and 1oDA based PHUs synthesized through different processes: $\mathrm{PHU}_{2}$ (reactive extrusion), $\mathrm{PHU}_{4}$ (magnetic stirring) and $\mathrm{PHU}_{6}$ (mechanical stirring) 


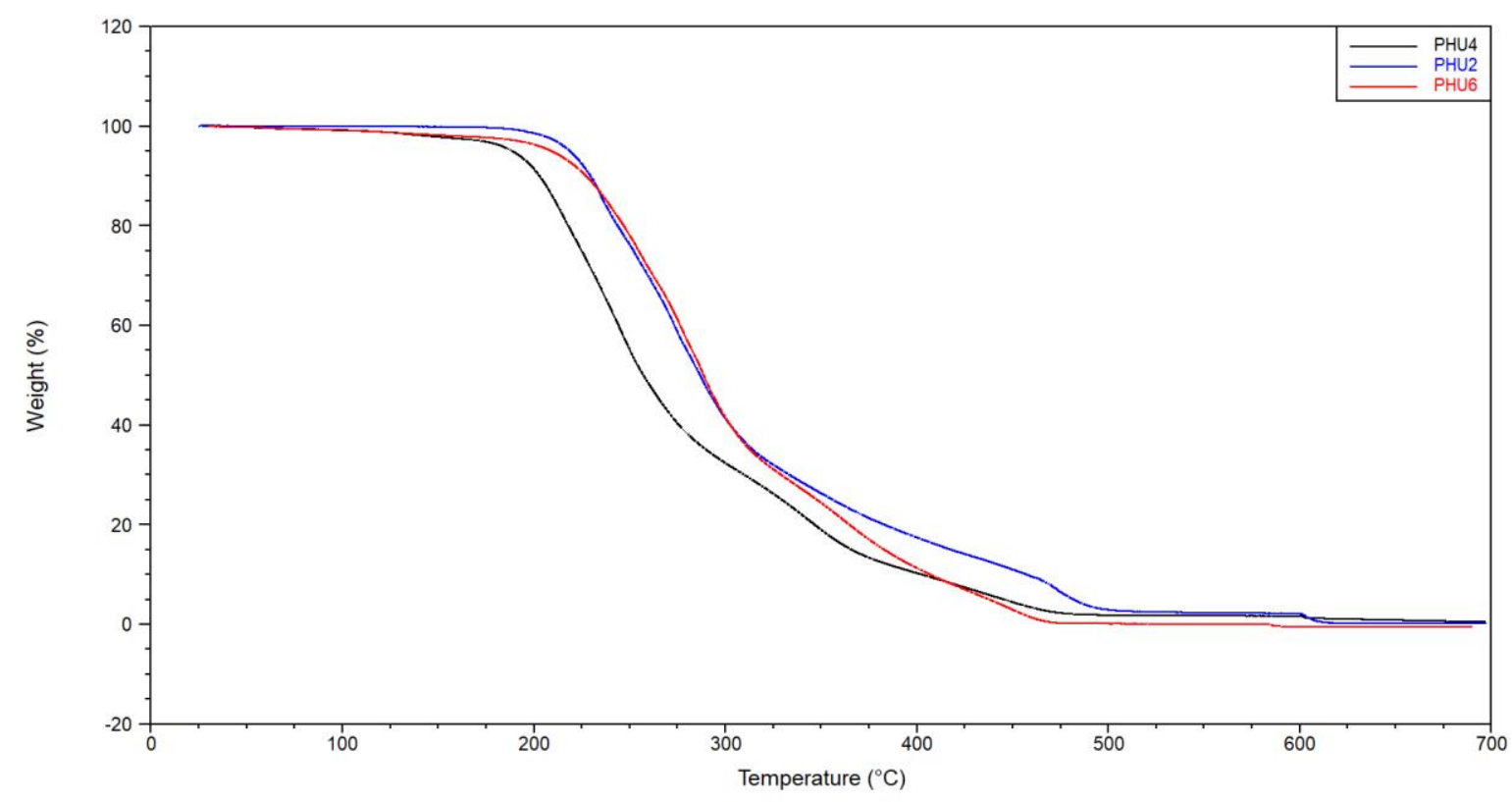

Figure SI-8. Overlay of TGA traces of DGDC/1oDA-based PHUs, synthesized through magnetic stirring $\left(\mathrm{PHU}_{4}\right.$, black), mechanical stirring $\left(\mathrm{PHU}_{6}\right.$, red $)$ and reactive extrusion $\left(\mathrm{PHU}_{2}\right.$, blue)

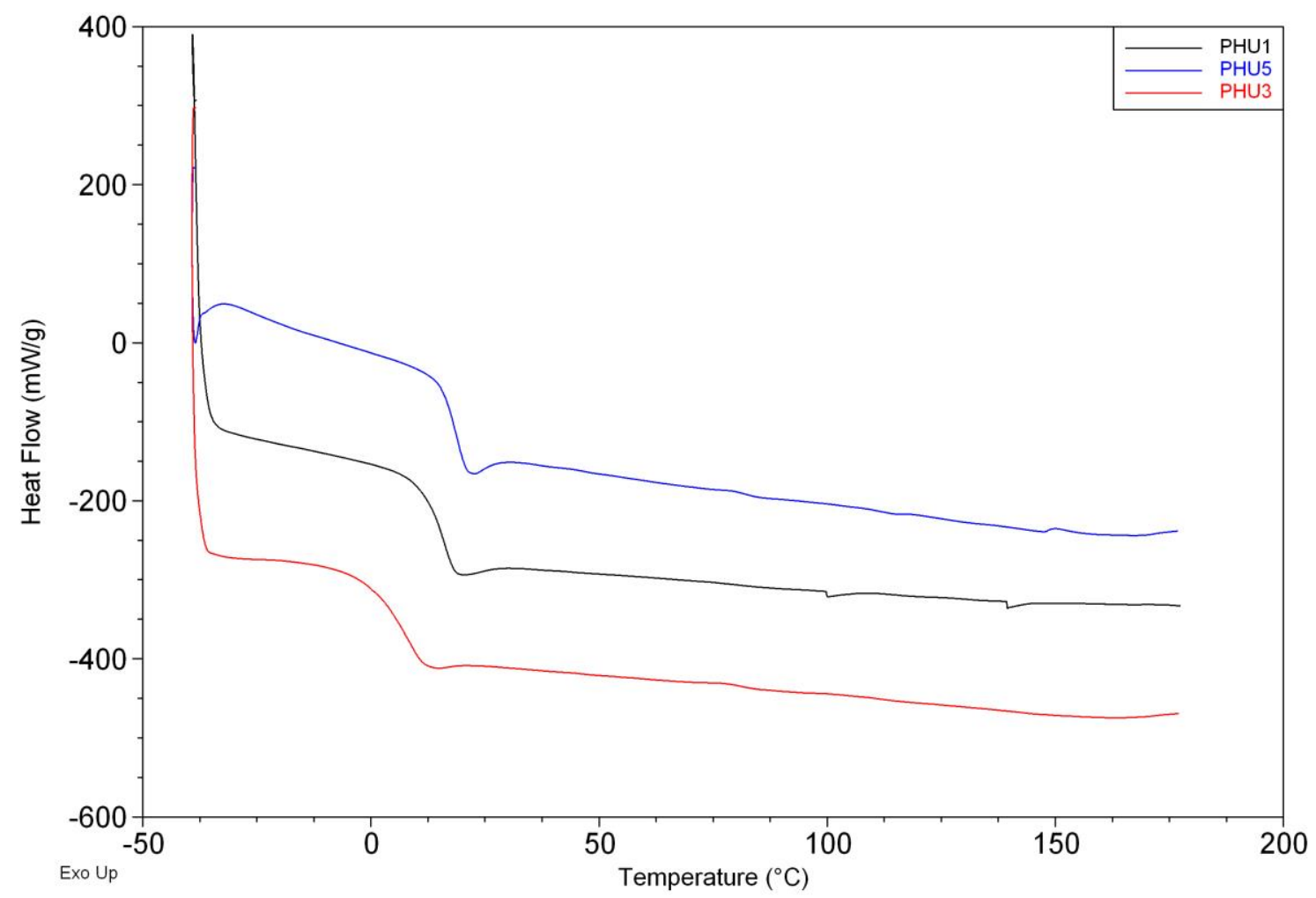

Figure SI-9. Overlay of DSC second heating cycle of DGDC/6DA-based PHUs. PHU1 (reactive extrusion, black), $\mathrm{PHU}_{3}$ (magnetic stirring, red), $\mathrm{PHU}_{5}$ (mechanical stirring, blue) 


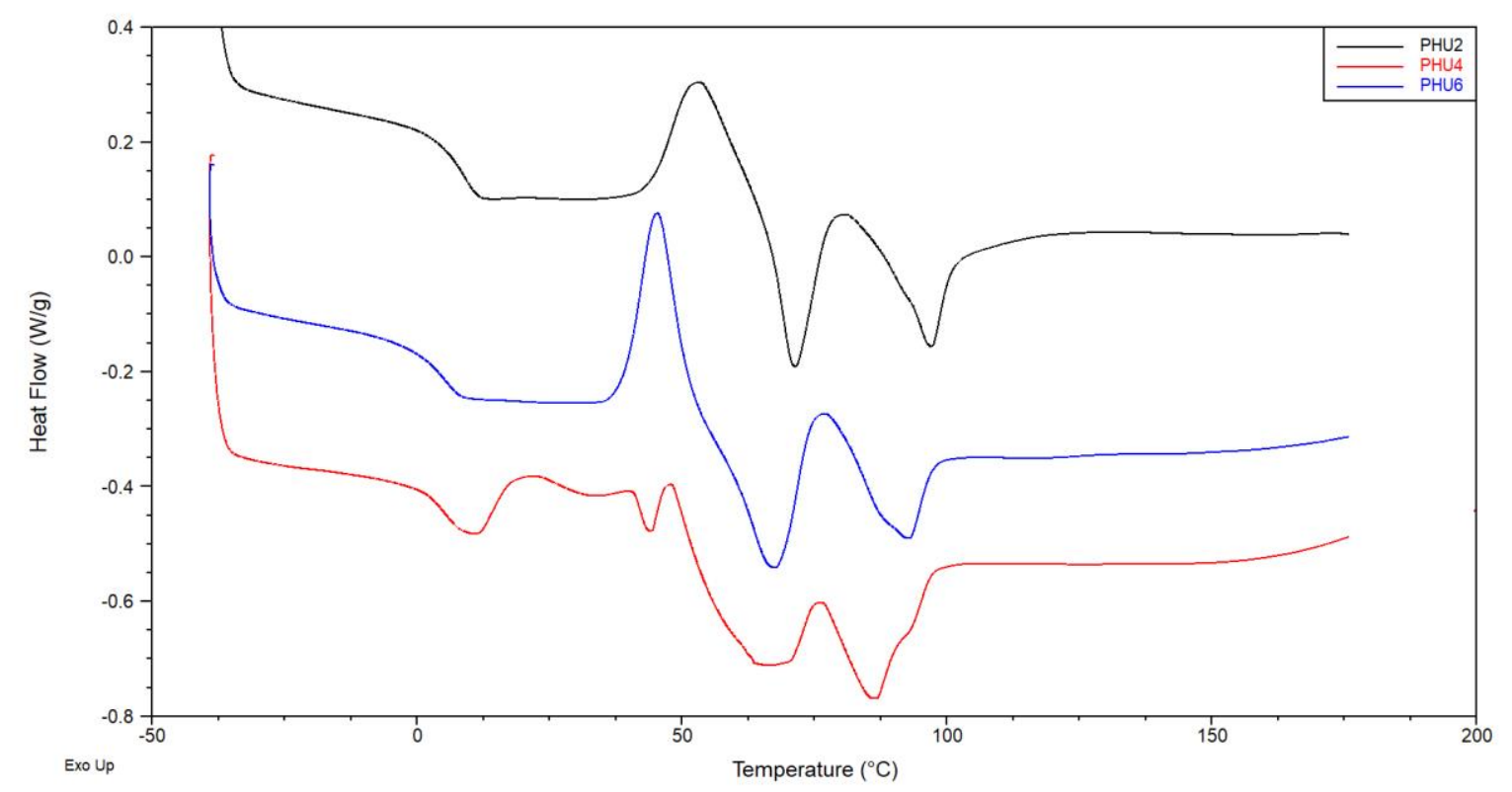

Figure SI-10. Overlay of DSC second heating cycle of DGDC/1oDA-based $\mathrm{PHUs}$. $\mathrm{PHU}_{2}$ (reactive extrusion, black), $\mathrm{PHU}_{4}$ (magnetic stirring, red), $\mathrm{PHU}_{6}$ (mechanical stirring, blue)

\section{Seb-bCC-ester based PHUs}
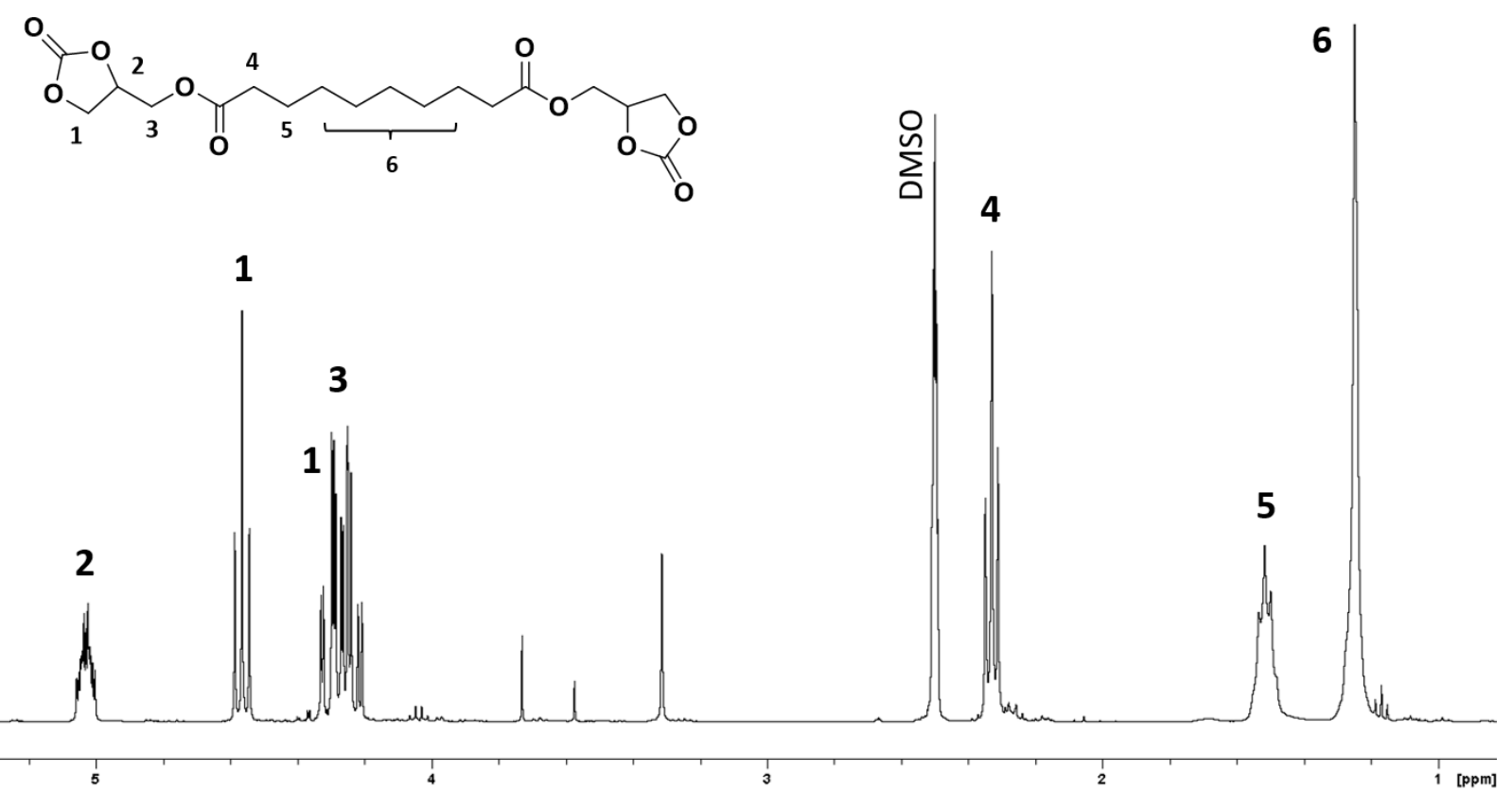

Figure SI-11. 'H NMR spectrum of Seb-bCC-ester, in DMSO-d6, RT, 64 scans 
A. Seb-bCC-ester based PHUs from reactive extrusion

Seb-bCC-ester based PHUs synthesized from reactive extrusion were found to be partially insoluble in classical solvents such as DMSO, leading to a soluble fraction (analyzed by classical NMR analysis) and to an insoluble fraction, which swells in DMSO-d6 and which can be characterized by the HR-MAS technique. The analysis was performed as follow:

The HR-MAS analysis was performed by the CESAMO (ISM-UMR5255, Universite de Bordeaux). The polymer was dissolved in small amount of DMSO and $20 \mu \mathrm{L}$ of the solution was introduced into a HRMAS-NMR $4 \mathrm{~mm}$ zirconia rotor, fitted with a perforated Teflon insert and sealed with a Kel-F cap. All NMR experiment were recorded at $25{ }^{\circ} \mathrm{C}$ on Bruker Avance III 600 equipped with a Bruker ${ }^{1} \mathrm{H}-{ }^{13} \mathrm{C}$ HRMAS probe working at ${ }^{13} \mathrm{C}$ and ${ }^{1} \mathrm{H}$ frequencies of 150.93 and $600.16 \mathrm{MHz}$, respectively. The rotor was inserted into the HR-MAS probe and MAS rate of $2500 \mathrm{~Hz}$. All NMR spectra were phased and baseline-corrected.

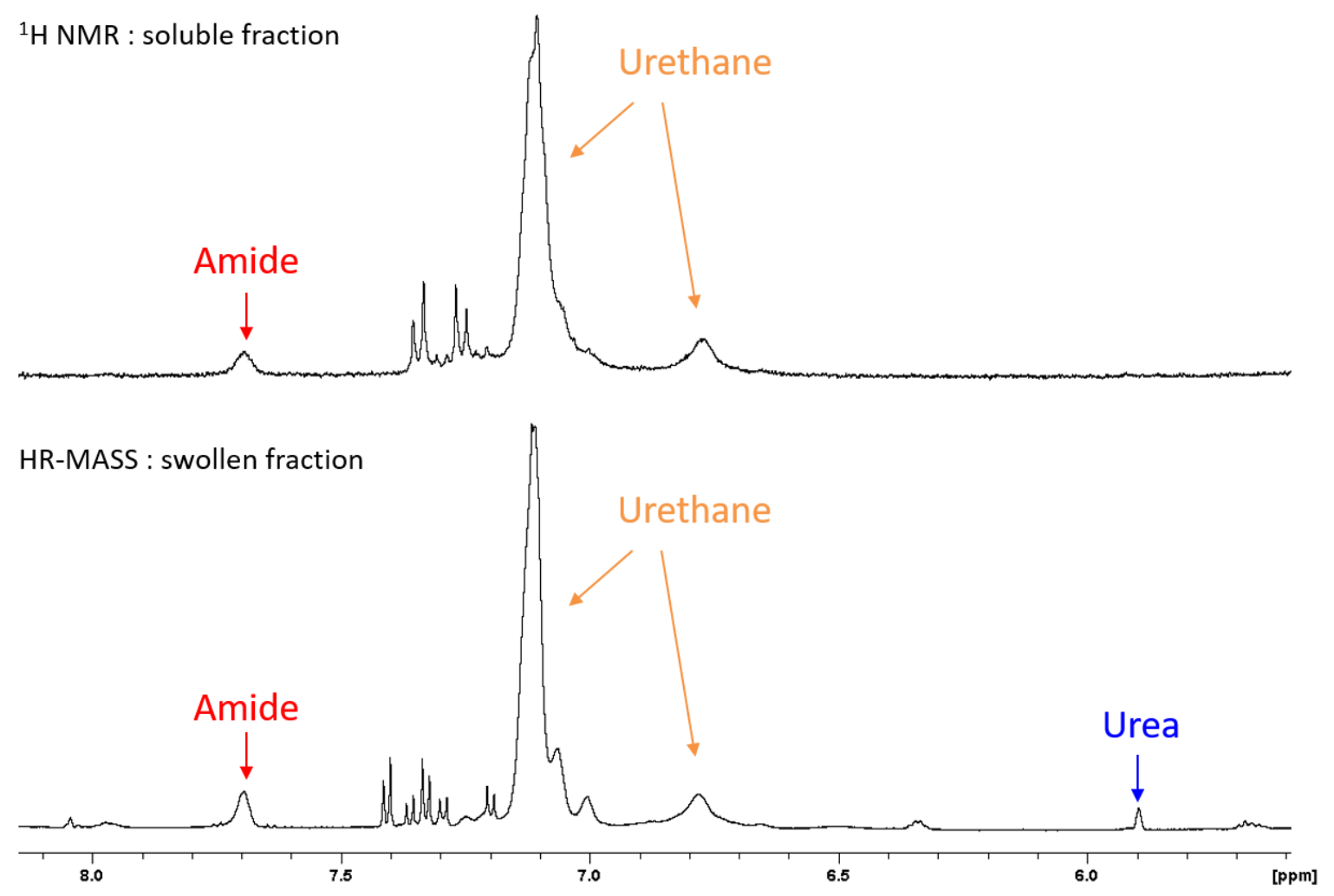

Figure SI-12. Zoom between 5.6 and 8.1ppm on ${ }^{1} \mathrm{H}$ NMR spectrum, of the soluble fraction of PHU ${ }_{10}$ in DMSOd6 at RT (top), Zoom between 5.6 and 8.1ppm on ${ }^{1} \mathrm{H}$ HR-MAS spectrum, of the insoluble and swollen fraction of PHU ${ }_{10}$ in DMSO-d6 at RT (bottom) 


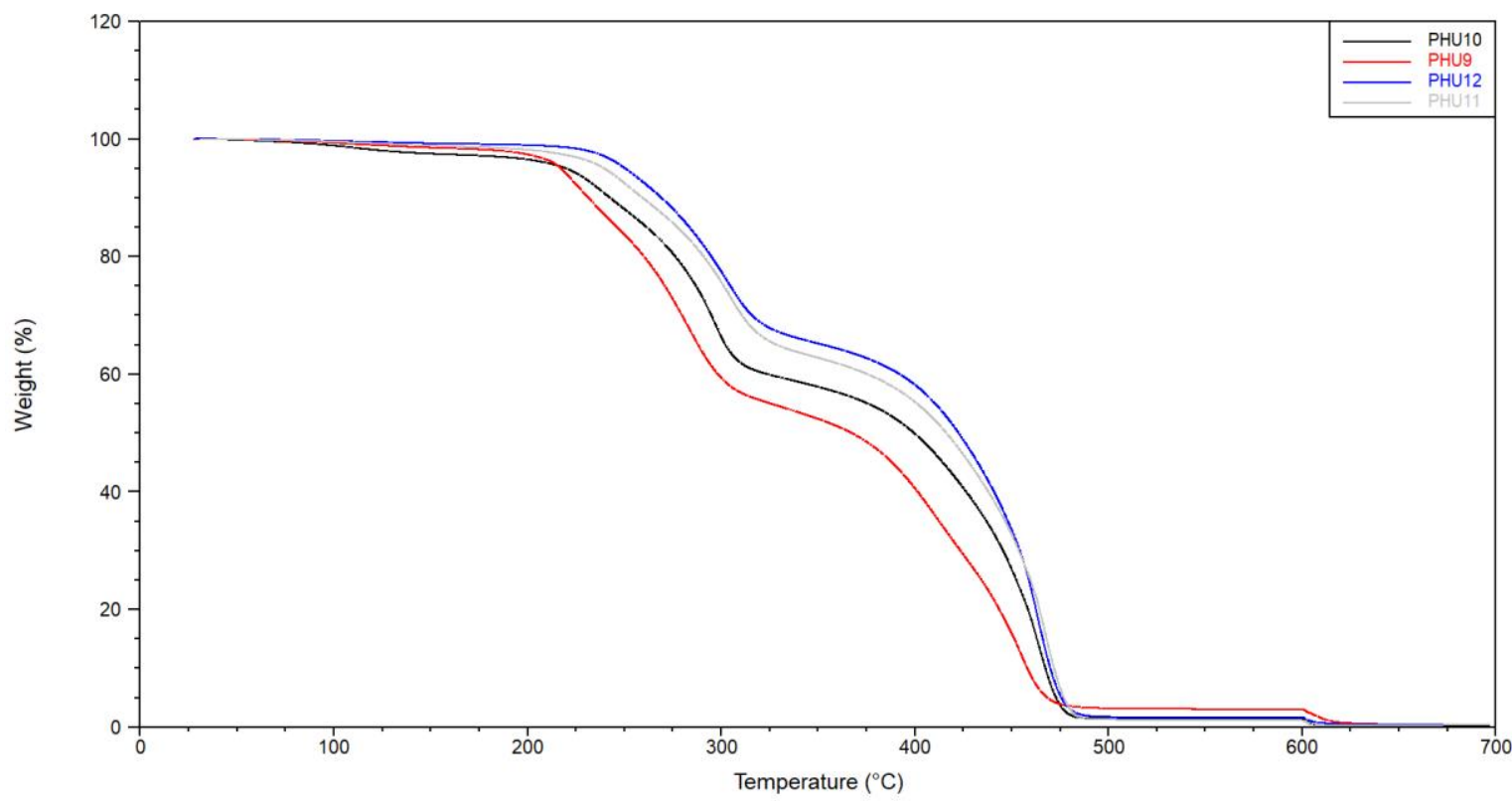

Figure SI-13. Overlay of TGA traces of Seb-bCC-ester-based PHUs synthesized through reactive extrusion.

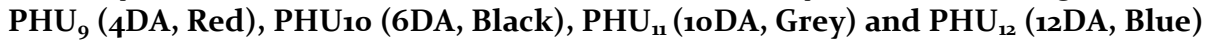

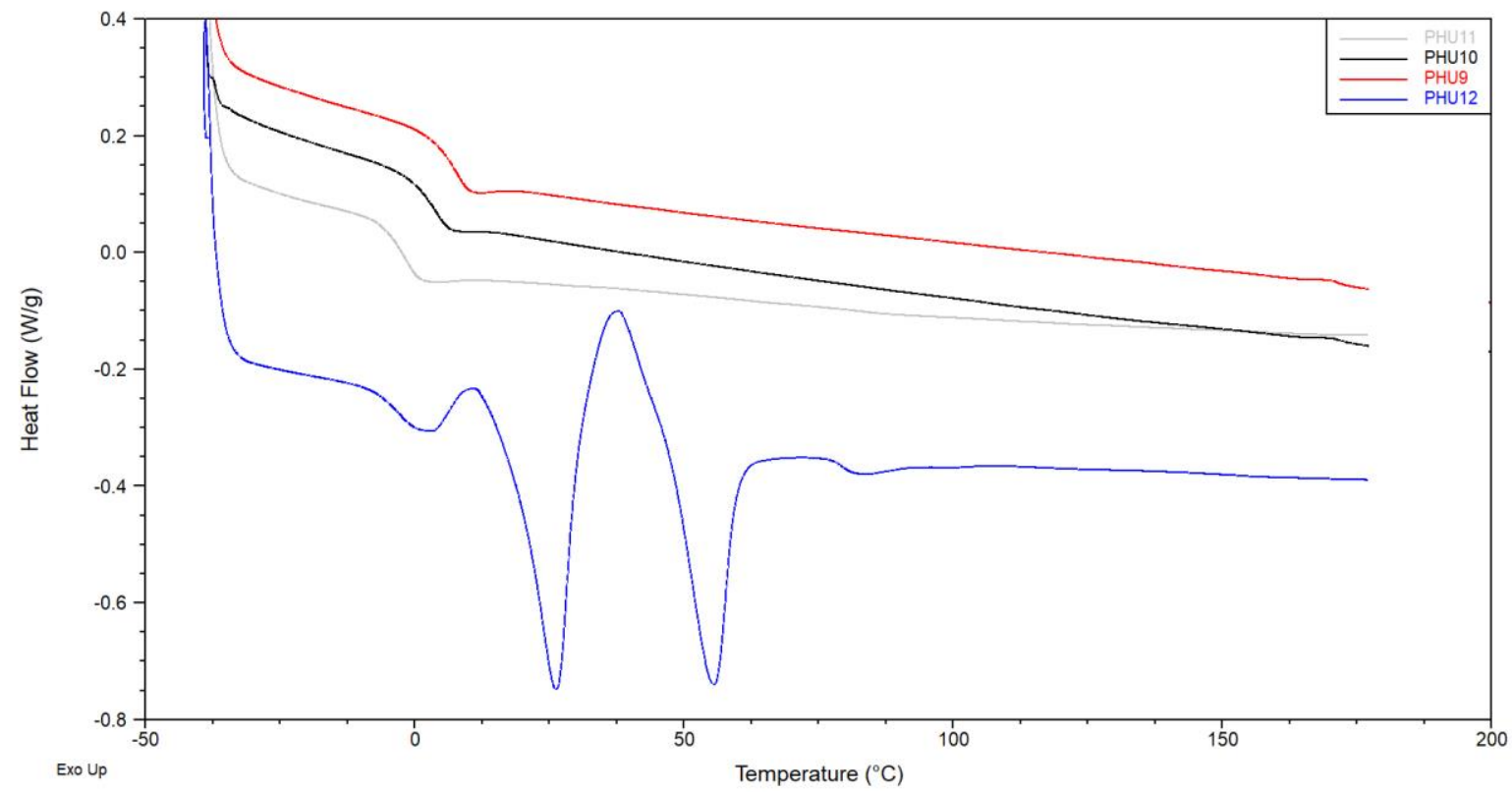

Figure SI-14. Overlay of TGA traces of Seb-bCC-ester-based PHUs synthesized through reactive extrusion $\mathrm{PHU}_{9}$ (Red), $\mathrm{PHU}_{10}$ (Black), $\mathrm{PHU}_{11}$ (Grey) and $\mathrm{PHU}_{12}$ (Blue) 
B. From magnetic and mechanical stirring

In order to compare reactive extrusion and classical polymerization stirring devices, four Seb-bCC-ester based PHUs were synthesized with 10DA and 6DA, through mechanical stirring and magnetic stirring. Synthesis details and associated thermal properties are summarized in table 1.

Seb-bCC-ester based PHUs synthesized with mechanical stirring were found to be soluble in classical solvents such as DMSO or DMF, making possible NMR and SEC analysis. Results are presented in figure 10 and 11. Given the shape of the chromatograms, meaning the spreading of the curves on the whole retention time scale, $M_{n}$ values were not calculated. Indeed, we are firmly convinced that those values, added to the dispersities, integrated on the so broad scale and would have been meaningless.

Table SI-1. Seb-bCC-ester-based PHUs synthesized from 6DA and 10DA, through mechanical and magnetic stirring, and associated thermal properties

\begin{tabular}{l|lllll} 
PHU & Process & Diamine & Duration & $T_{g}\left({ }^{\circ} \mathrm{C}\right)$ & $T_{d 5 \%}$ \\
\hline 13 & Magnetic Stirring & 6DA & $1 \mathrm{~h}$ & -9.5 & 235 \\
14 & Mechanical Stirring & 6DA & 1h & -2.5 & 242 \\
19 & Magnetic Stirring & 10DA & 21min & -3.3 & $\mathrm{Nd}$ \\
20 & Mechanical Stirring & 10DA & $21 \mathrm{~min}$ & -3.3 & 246
\end{tabular}
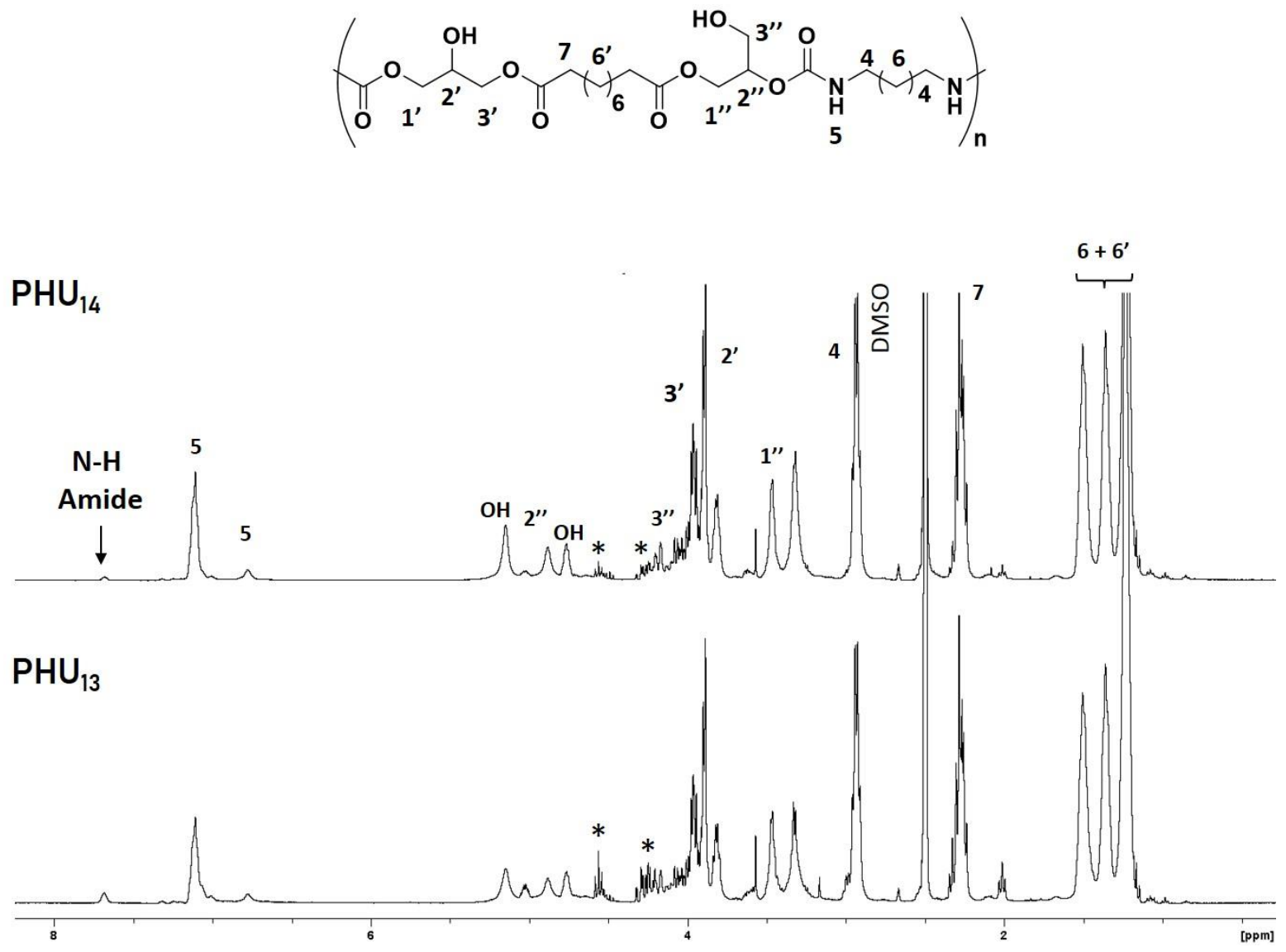

Figure SI-15. ${ }^{1} \mathrm{H}$ NMR spectrum of PHU14, Seb-bCC-ester/6DA/Mechanical stirring (top) and magnetic stirring (bottom), in DMSO-d6, RT, 64 scans. *Residual cyclic carbonate. Ratio Amide/Urethane/Urea Conversion: 1.7/98.3/o- 89.1\% $\left(\mathrm{PHU}_{14}\right)$, 5.9/94.1/o - 76.5\% $\left(\mathrm{PHU}_{13}\right)$. 


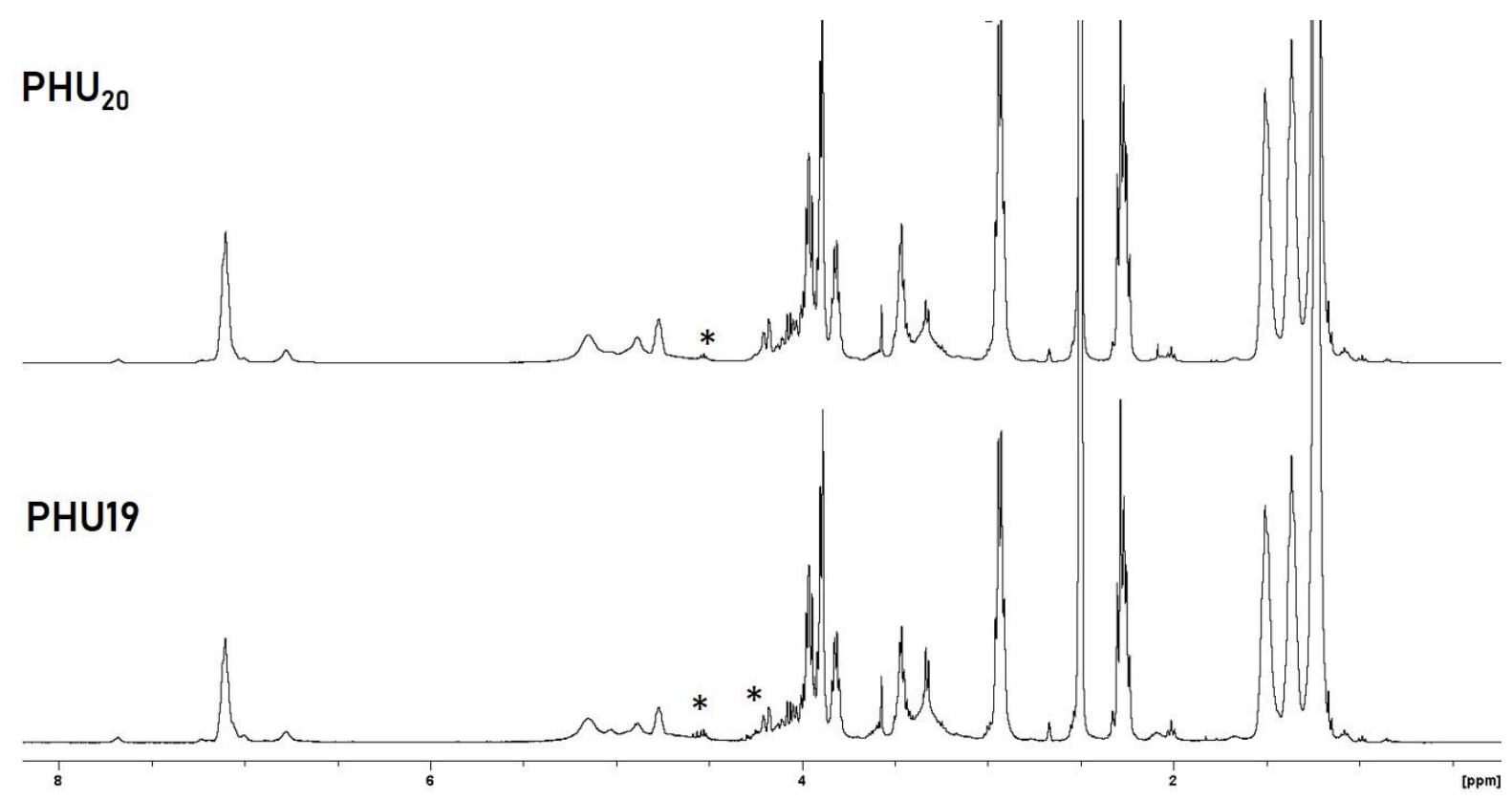

Figure SI-16. 'H NMR spectrum of PHU19, Seb-bCC-ester/1oDA/Mechanical stirring (top) and magnetic stirring (bottom), in DMSO-d6, RT, 64 scans. * Residual cyclic carbonate. Ratio Amide/Urethane/Urea -

Conversion: 2.2/97.8/o - 85.9\% $\left(\mathrm{PHU}_{19}\right)$, 1.7/98.3/o - 90.0\%

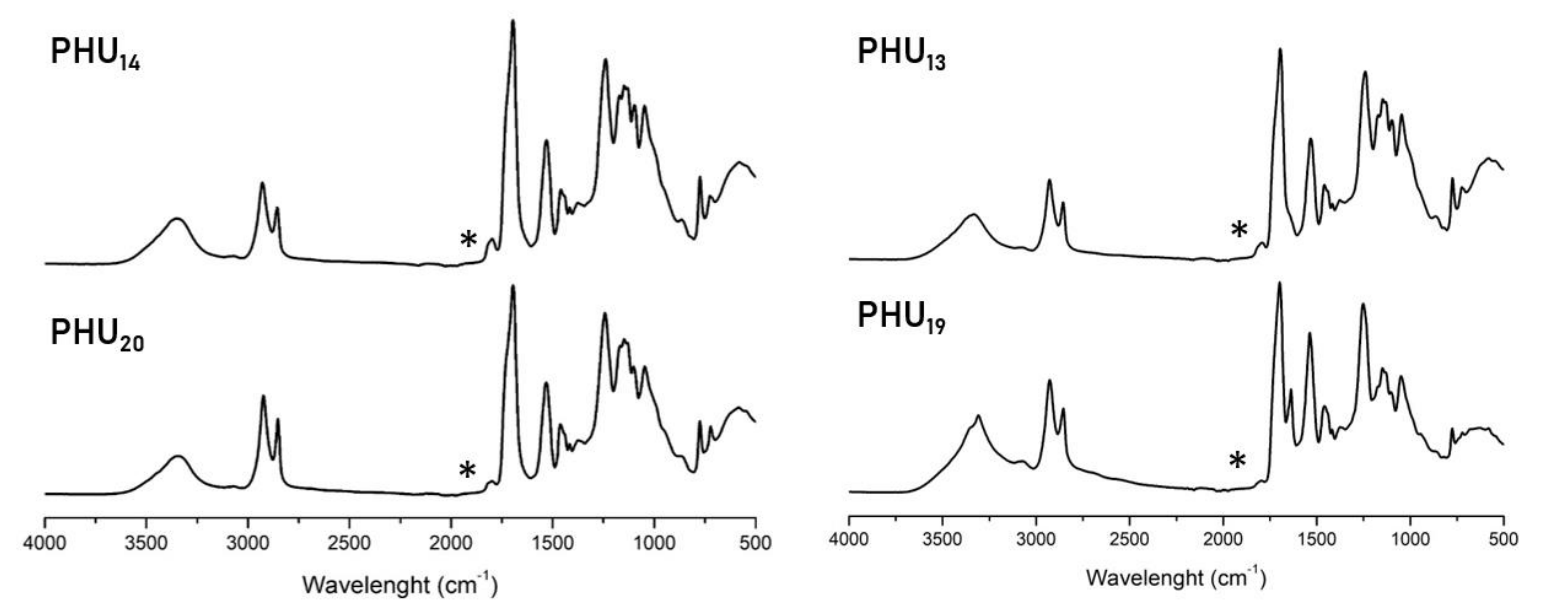

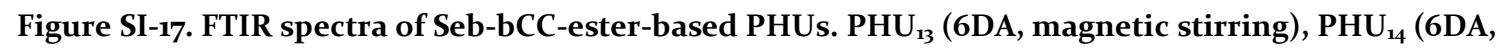
mechanical stirring), $\mathrm{PHU}_{19}$ (10DA, magnetic stirring) and $\mathrm{PHU}_{20}$ (10DA, mechanical stirring). 


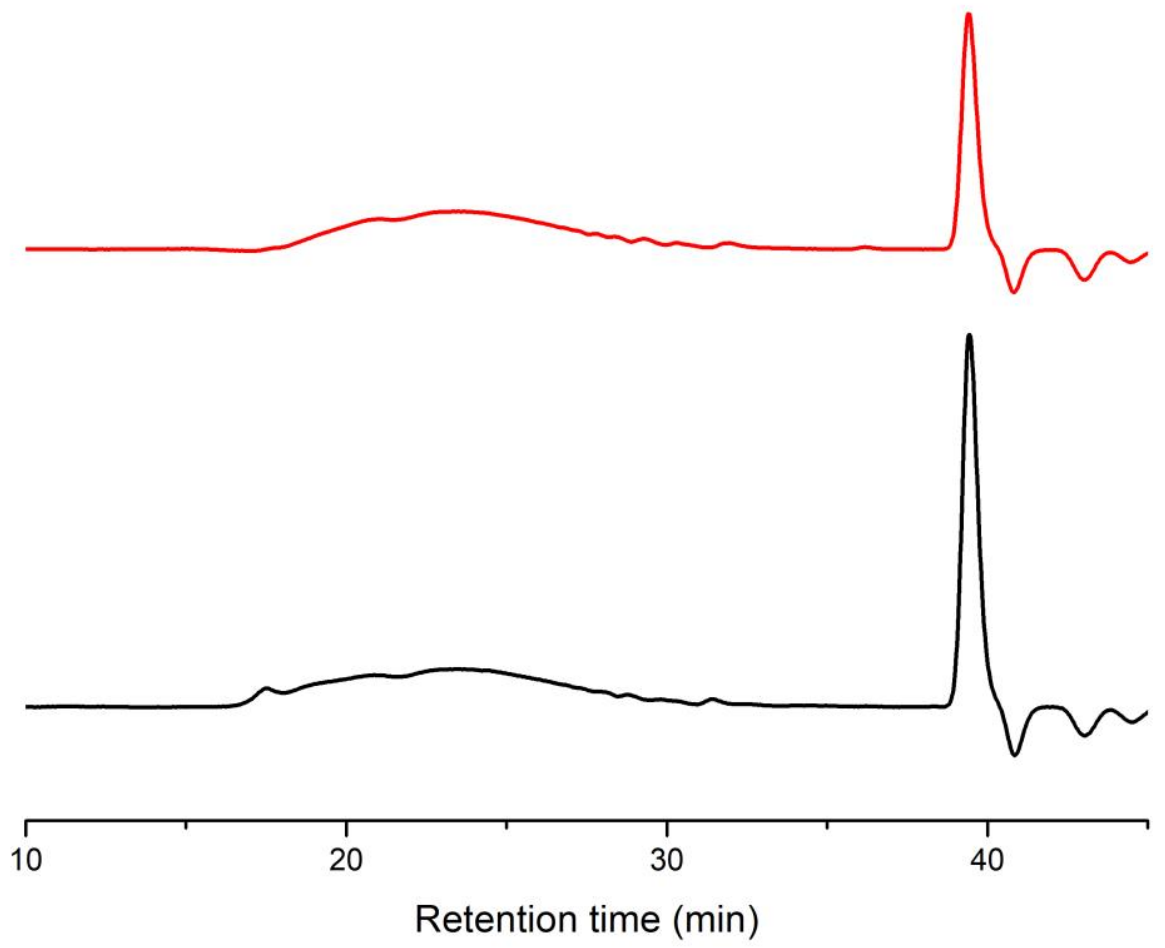

Figure SI-18. SEC traces in DMF of Seb-bCC-ester-based PHUs made through mechanical stirring from 6DA ( $\mathrm{PHU}_{13}$, top), and 1oDA ( $\mathrm{PHU}_{19}$ bottom) 


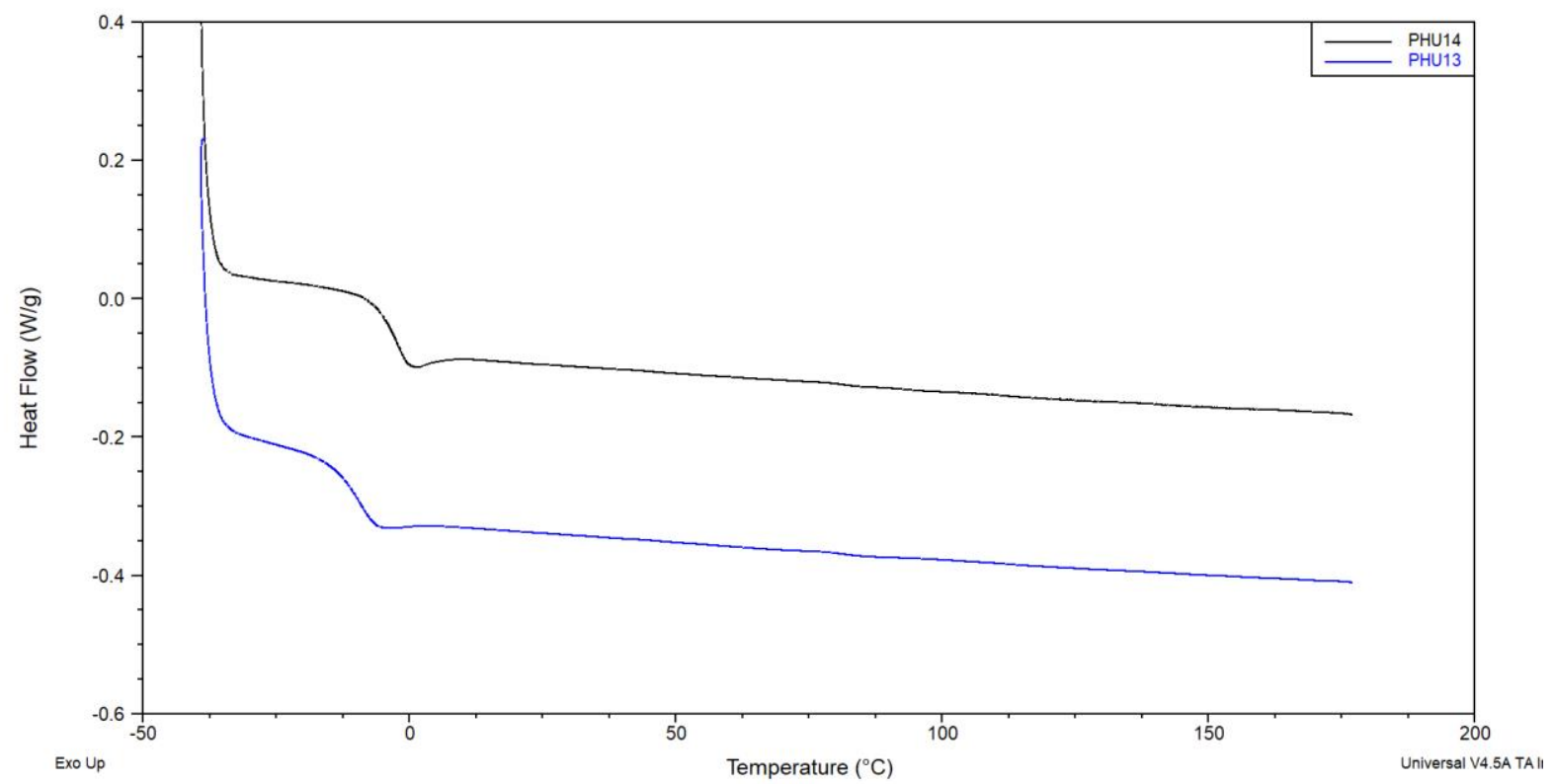

Figure SI-19. Overlay of DSC second heating cycles of $\mathrm{PHU}_{13}$ (Seb-bCC-ester/6DA, magnetic stirring) and $\mathrm{PHU}_{14}$ (Seb-bCC-ester/6DA, mechanical stirring)

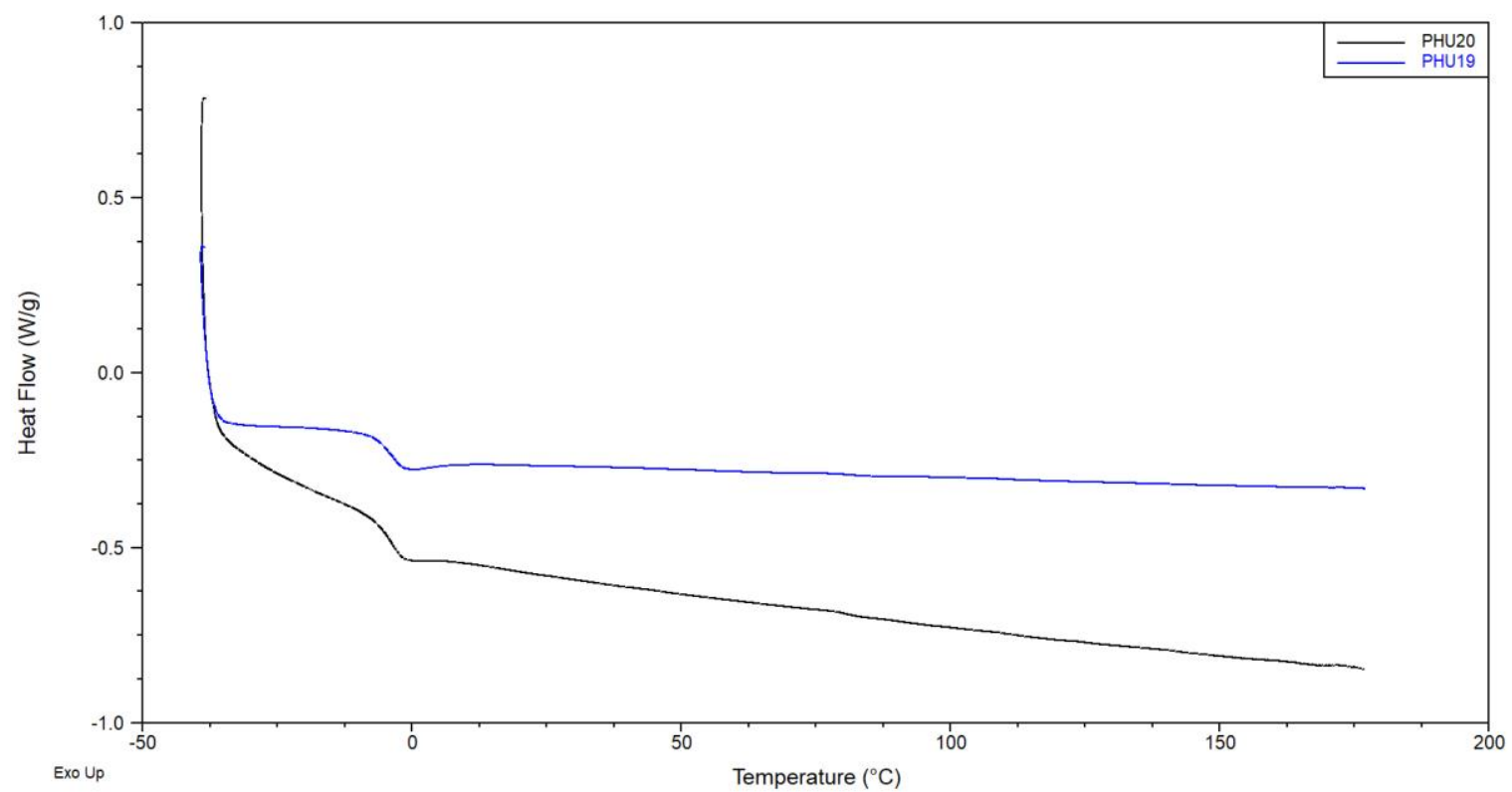

Figure SI-2o. Overlay of DSC second heating cycle of PHU19 (Seb-bCC-ester/1oDA, magnetic stirring) and PHU2o (Seb-bCC-ester/1oDA, mechanical stirring) 


\section{Und-6DA-based PHUs}

\section{Und-6DA}
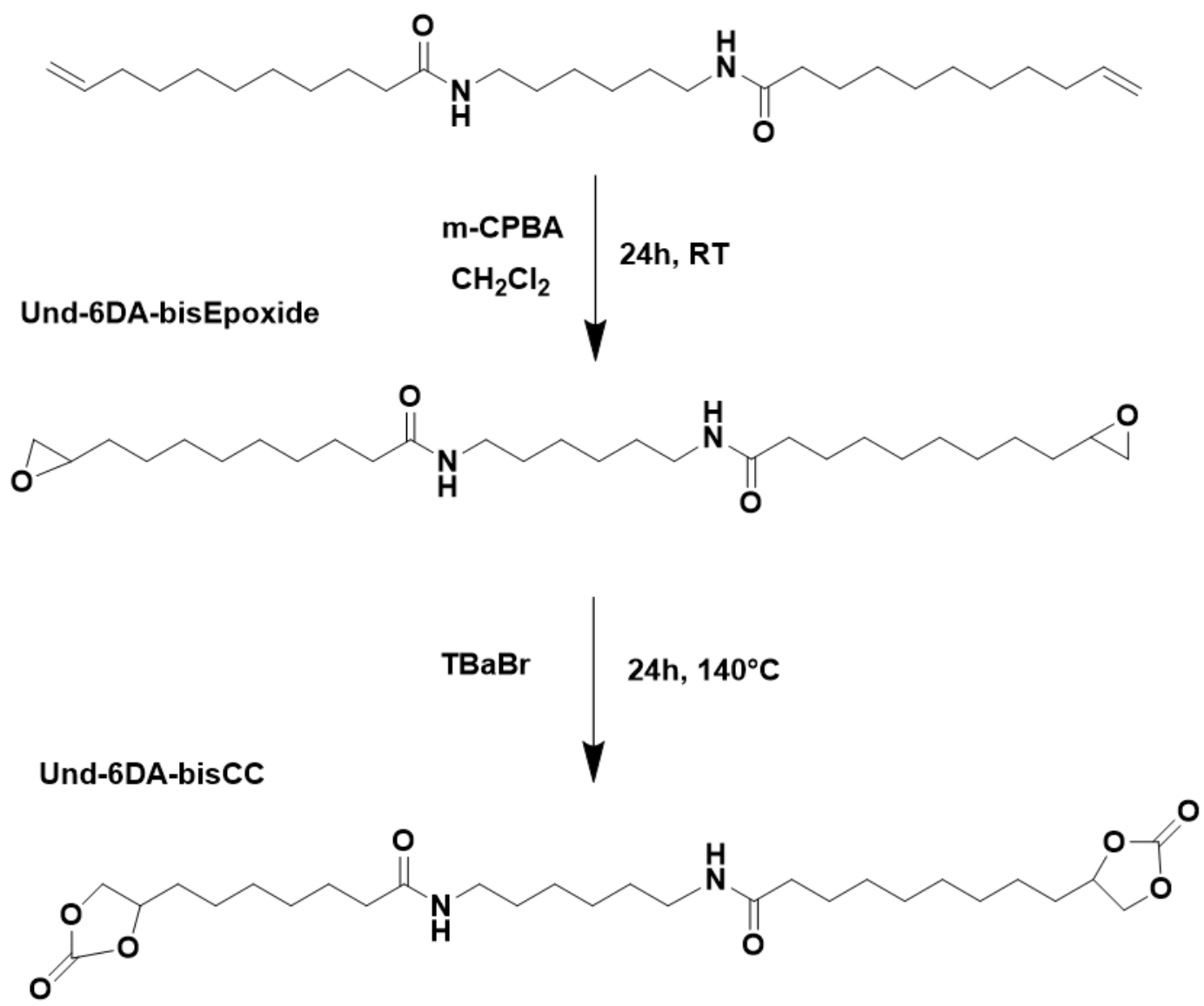

Figure SI-21. Epoxidation and carbonation steps leading to Und-DA-bisCC from Und-6DA 


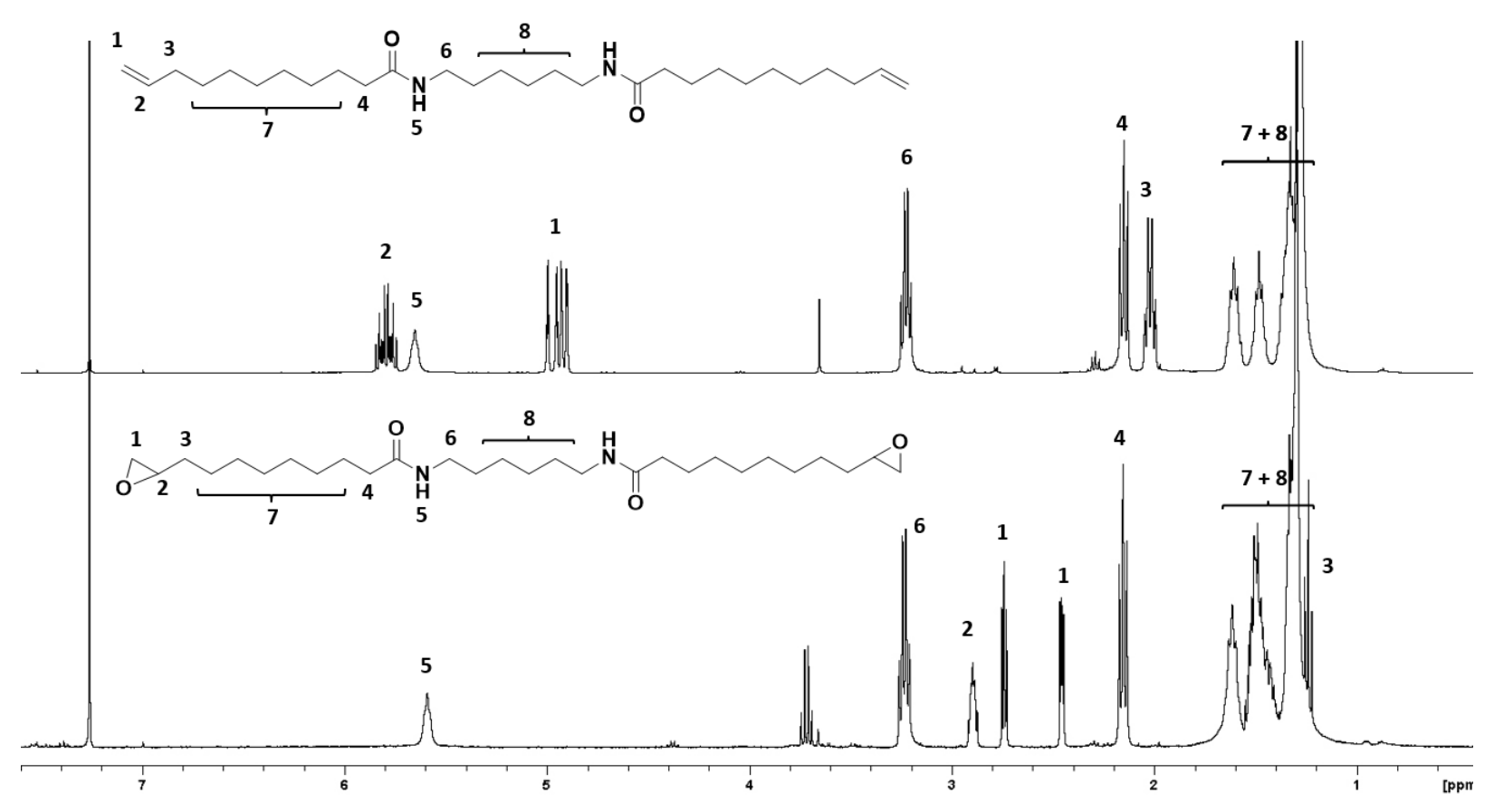

Figure SI-22. Stacked ${ }^{1} \mathrm{H}$ NMR Spectra of Und-6DA (top) and Und-6DA-bisEpoxyde (bottom), in $\mathrm{CDCl}_{3}, \mathrm{RT}$

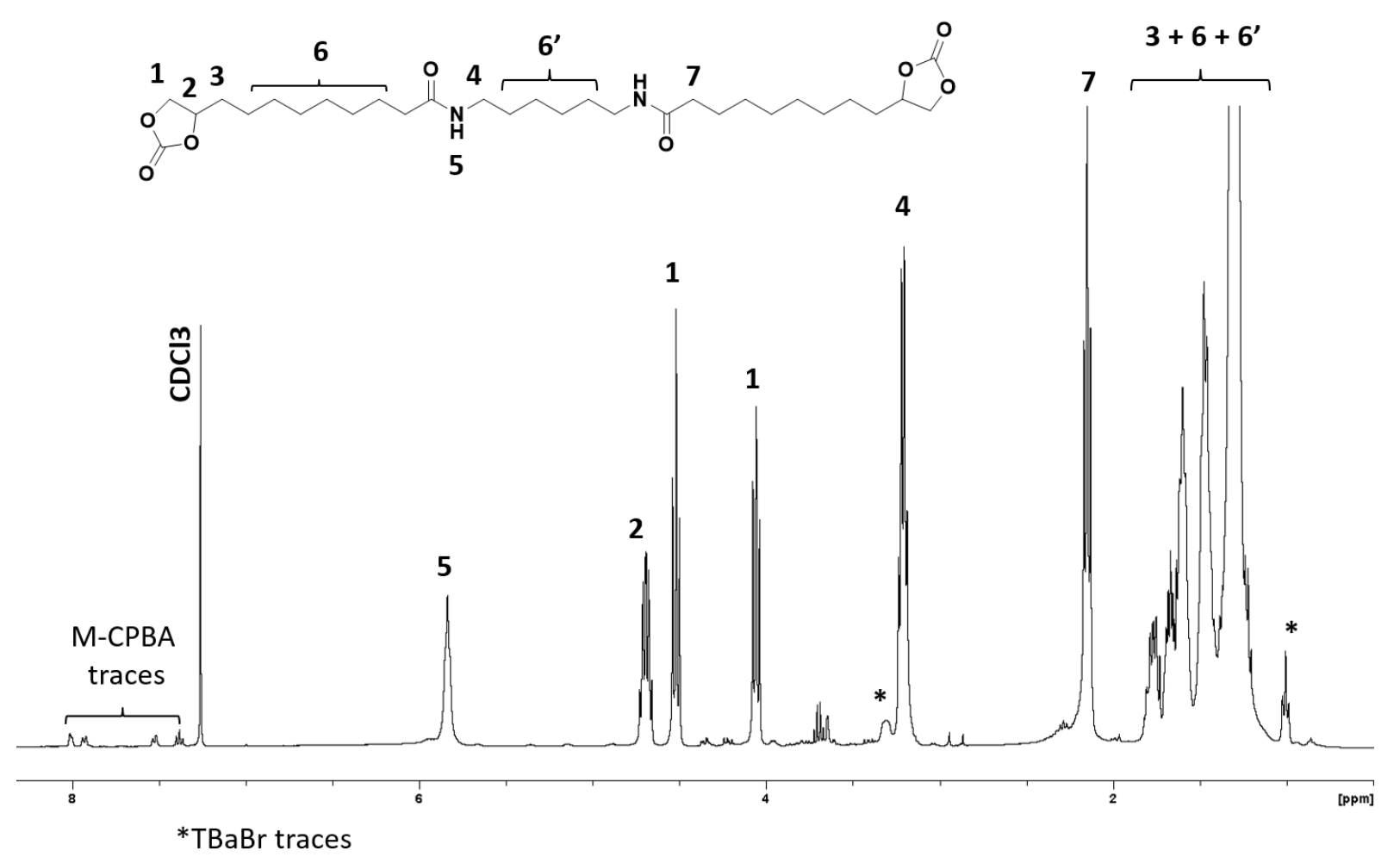

Figure SI-23. ${ }^{1} \mathrm{H}$ NMR spectrum of Und-6DA-bisCC in $\mathrm{CDCl}_{3}$, $\mathrm{RT}, 6_{4}$ scans. ${ }^{*} \mathrm{TBaBr}$ traces 

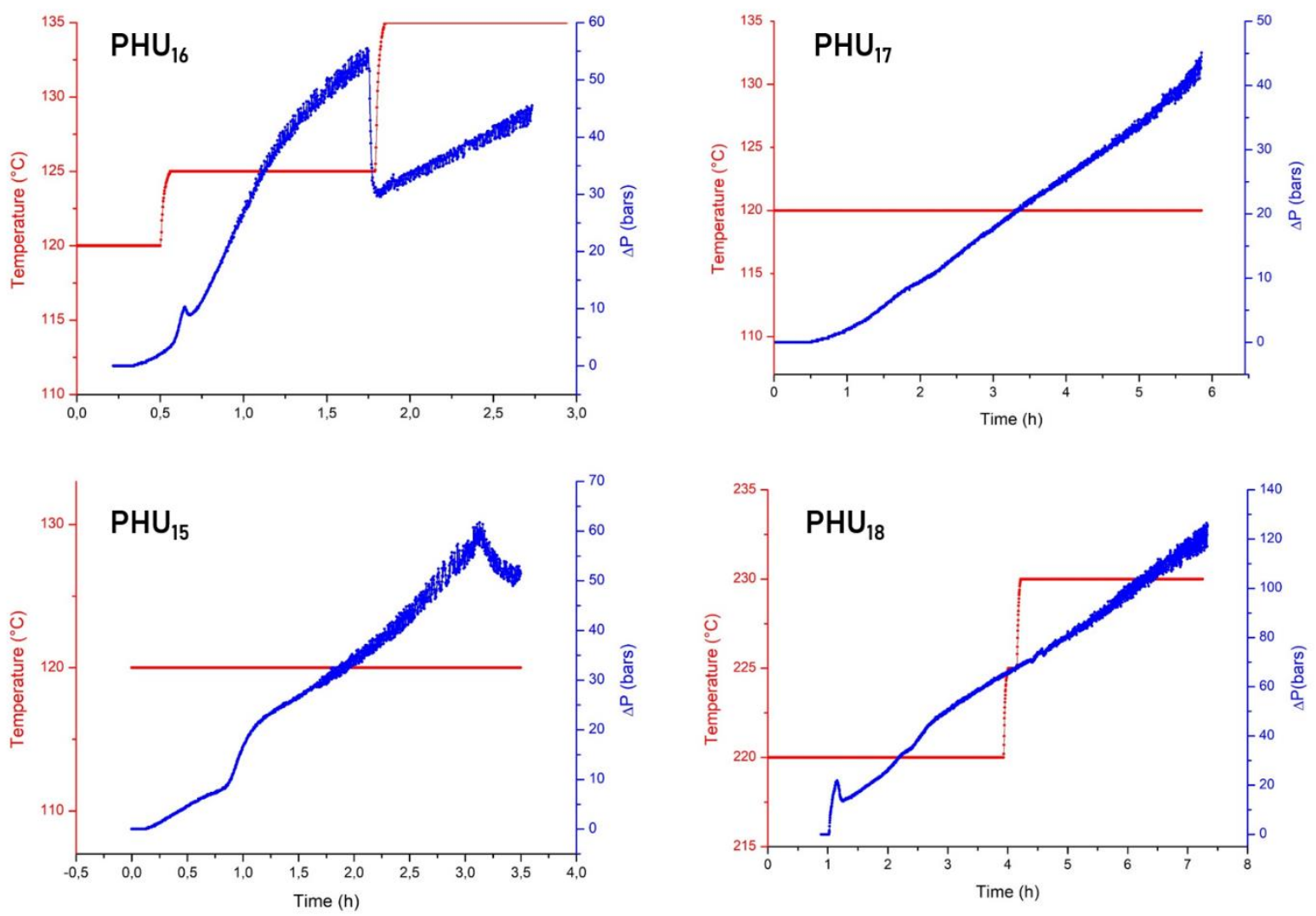

Figure SI-24. $\Delta P=f(t)$ profiles obtained during synthesis of Und-6DA-based PHUs. Top: PHU 16 (6DA, left), PHU PHxylylène-DA, right). Bottom: PHU15 (1oDA, left), $\mathrm{PHU}_{18}$ (L-Lysine, right) 


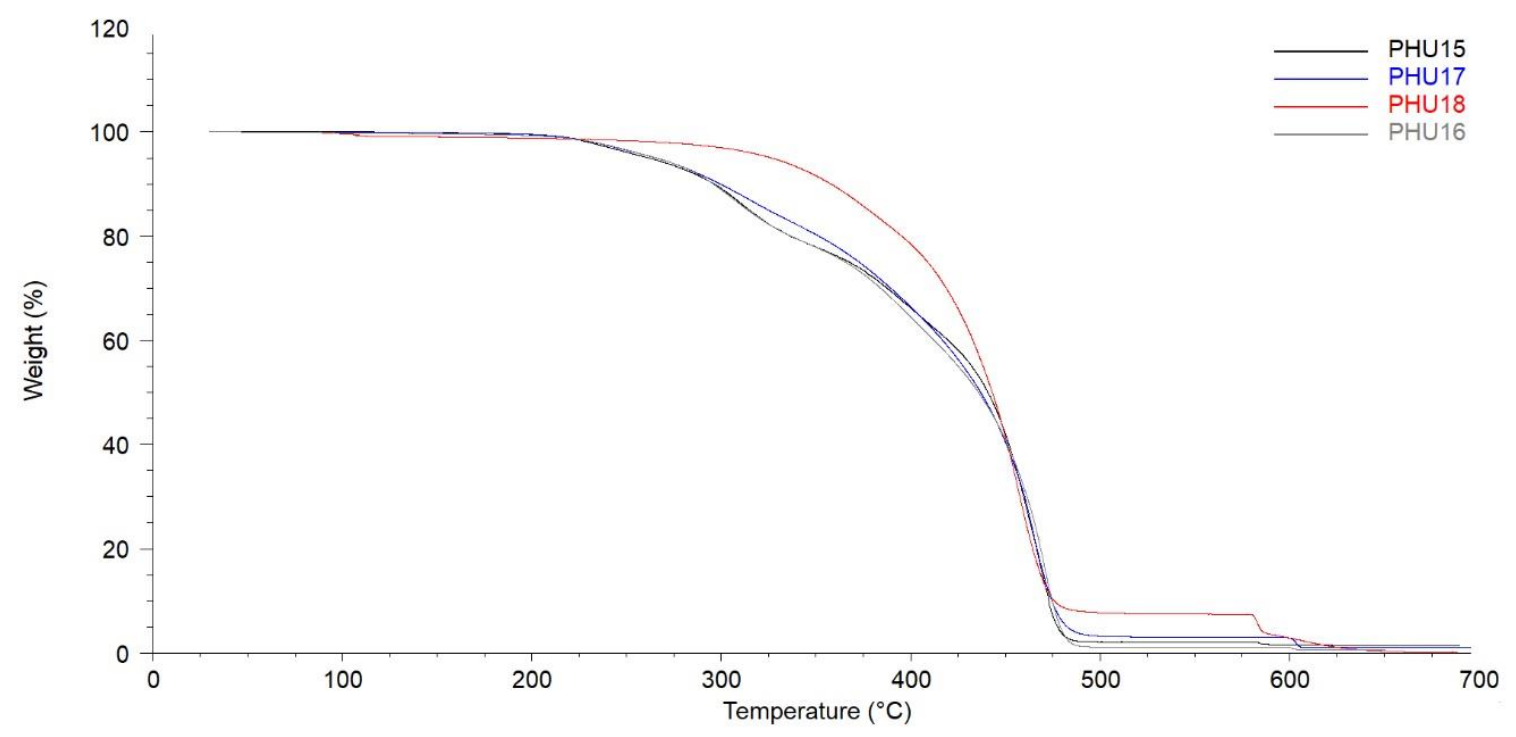

Figure SI-25. Overlay of TGA traces of Und-6DA-bisCC-based PHUs, $10^{\circ} \mathrm{C} / \mathrm{min}$, under azote until $600^{\circ} \mathrm{C}$ and under air until $700^{\circ} \mathrm{C}$.

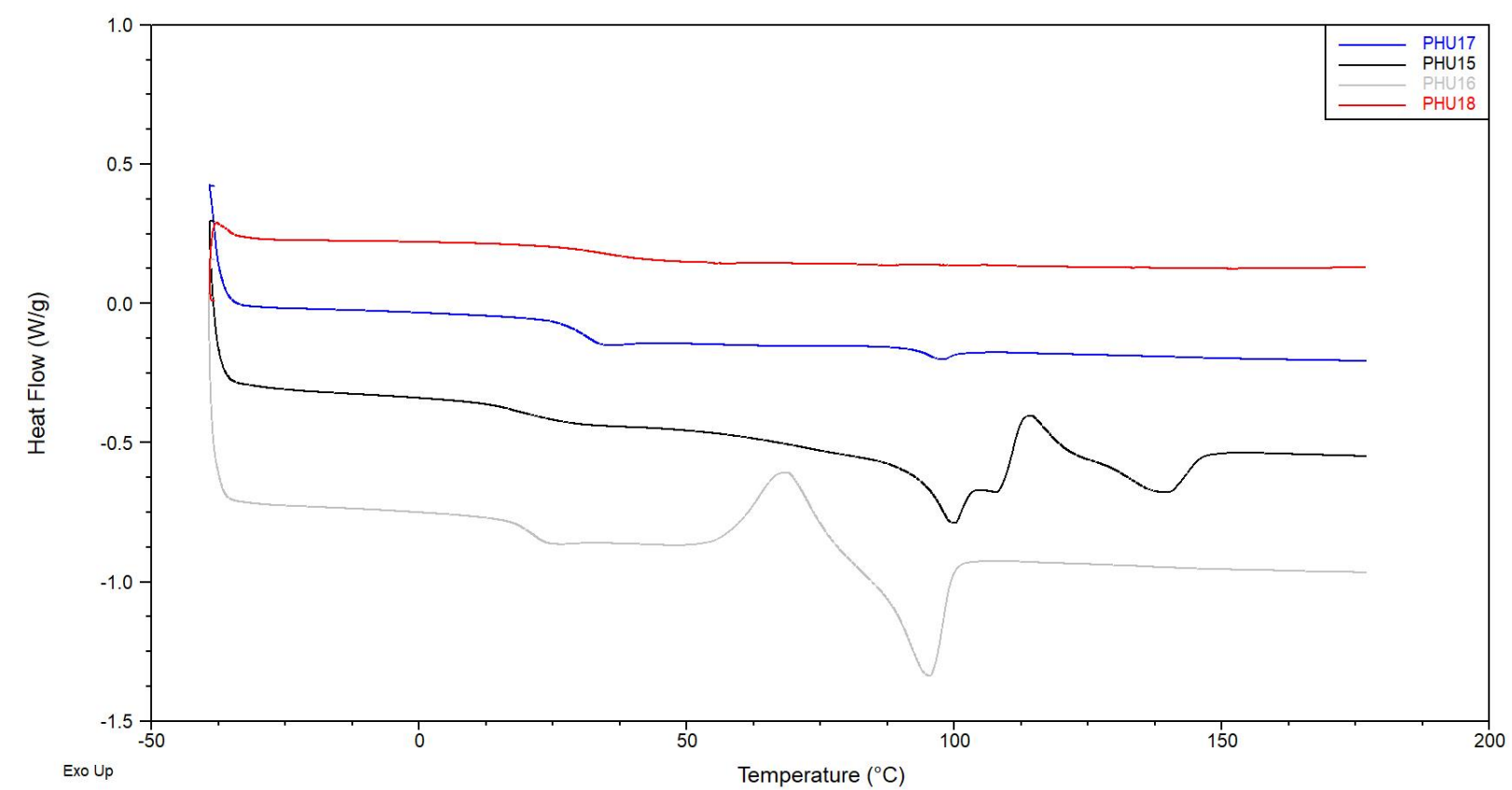

Figure SI-26. Overlay of DSC second heating cycles of Und-6DA-bisCC-based PHUs, $10^{\circ} \mathrm{C} / \mathrm{min}$, from $-40^{\circ} \mathrm{C}$ to $180^{\circ} \mathrm{C}$. 